\title{
Genomic and transcriptomic analysis unveils population evolution and development of pesticide resistance in fall armyworm Spodoptera frugiperda
}

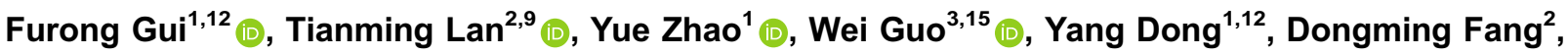
Huan Liu ${ }^{2,9}$ (i), Haimeng $\mathrm{Li}^{2}$, Hongli Wang ${ }^{2}$, Ruoshi Hao ${ }^{12}$, Xiaofang Cheng ${ }^{5}$, Yahong $\mathrm{Li}^{16}$,

Pengcheng Yang ${ }^{4}(\mathbb{0})$, Sunil Kumar Sahu ${ }^{2}$ (), Yaping Chen ${ }^{1}$, Le Cheng ${ }^{7}$, Shuqi He ${ }^{1}$, Ping Liu ${ }^{5}$, Guangyi Fan ${ }^{6}$, Haorong $\mathrm{Lu}^{8,10}$, Guohai $\mathrm{Hu}^{8,10}$, Wei Dong ${ }^{2}$, Bin Chen ${ }^{1}$, Yuan Jiang ${ }^{18}$, Yongwei Zhang ${ }^{18}$, Hanhong $\mathrm{Xu}^{17}$, Fei Lin ${ }^{17}$, Bernard Slippers ${ }^{19}$, Alisa Postma ${ }^{19}$, Matthew Jackson ${ }^{19}$, Birhan Addisie Abate ${ }^{20}$, Kassahun Tesfaye ${ }^{20,21}$, Aschalew Lemma Demie ${ }^{20}$, Meseret Destaw Bayeleygne ${ }^{20}$, Dawit Tesfaye Degefu ${ }^{22}$, Feng Chen ${ }^{5}$, Paul K. Kuria ${ }^{23}$, Zachary M. Kinyua ${ }^{23}$, Tong-Xian Liu ${ }^{13}$, Huanming Yang ${ }^{10,11}$,

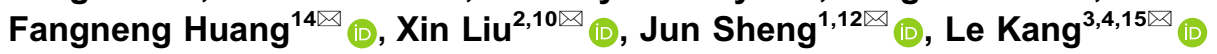

${ }^{1}$ State Key Laboratory for Conservation and Utilization of Bioresources in Yunnan, Yunnan Agricultural University, Kunming 650201, China

2 State Key Laboratory of Agricultural Genomics, BGI-Shenzhen, Shenzhen 518083, China

3 State Key Laboratory of Integrated Management of Pest Insects and Rodents, Institute of Zoology, Chinese Academy of Sciences, Beijing 100101, China

${ }^{4}$ Beijing Institutes of Life Science, Chinese Academy of Sciences, Beijing 100101, China

${ }^{5} \mathrm{MGI}, \mathrm{BGI}-S h e n z h e n$, Shenzhen 518083, China

${ }^{6}$ BGI-Qingdao, BGI-Shenzhen, Qingdao 266555, China

7 BGI-Yunnan, No. 389 Haiyuan Road, High-tech Development Zone, Kunming 650106, China

${ }^{8}$ China National GeneBank, Jinsha Road, Dapeng New District, Shenzhen 518120, China

${ }^{9}$ Department of Biology, University of Copenhagen, 2100 Copenhagen, Denmark

${ }^{10}$ Guangdong Provincial Key Laboratory of Genome Read and Write, BGI-Shenzhen, Shenzhen 518120, China

${ }^{11}$ Guangdong Provincial Academician Workstation of BGI Synthetic Genomics, BGI-Shenzhen, Shenzhen 518120, China

12 Yunnan Plateau Characteristic Agriculture Industry Research Institute, Kunming 650201, China

${ }^{13}$ College of Plant Health and Medicine, Qingdao Agricultural University, Qingdao 266109, China

${ }^{14}$ Department of Entomology, Louisiana State University Agricultural Center, Baton Rouge, LA 70803, USA

${ }^{15}$ CAS Center for Excellence in Biotic Interactions, University of Chinese Academy of Sciences, Beijing 100101, China

${ }^{16}$ Yunnan Plant Protection and Quarantine Station, Kunming 650034, China

17 State Key Laboratory for Conservation and Utilization of Subtropical Agro-Bioresources, South China Agricultural University, Guangzhou 510642, China

${ }^{18}$ BGI-Americas, One Broadway, 14th Floor, Cambridge, MA 02142, USA

19 Department of Biochemistry, Genetics and Microbiology, Forestry and Agricultural Biotechnology Institute, University of Pretoria, Pretoria, South Africa

${ }^{20}$ Ethiopian Biotechnology Institute, Addis Ababa, Ethiopia

${ }^{21}$ College of Natural Science, Addis Ababa University, Addis Ababa, Ethiopia

${ }^{22}$ Melkassa Agricultural Research Center, Ethiopian Institute of Agricultural Research, Melkassa, Addis Ababa, Ethiopia

${ }^{23}$ Kenya Agricultural and Livestock Research Organization, P.O. Box 57811, Nairobi 00800, Kenya

$凶$ Correspondence: FHuang@agcenter.Isu.edu (Fangneng Huang), liuxin@genomics.cn (X. Liu), shengjunpuer@163.com (J. Sheng), Ikang@ioz.ac.cn (L. Kang)

Received June 3, 2020 Accepted September 15, 2020 


\section{ABSTRACT}

The fall armyworm (FAW), Spodoptera frugiperda, is a destructive pest native to America and has recently become an invasive insect pest in China. Because of its rapid spread and great risks in China, understanding of FAW genetic background and pesticide resistance is urgent and essential to develop effective management strategies. Here, we assembled a chromosome-level genome of a male FAW (SFynMstLFR) and compared resequencing results of the populations from America, Africa, and China. Strain identification of 163 individuals collected from America, Africa and China showed that both $\mathrm{C}$ and $\mathrm{R}$ strains were found in the American populations, while only $C$ strain was found in the Chinese and African populations. Moreover, population genomics analysis showed that populations from Africa and China have close relationship with significantly genetic differentiation from American populations. Taken together, FAWs invaded into China were most likely originated from Africa. Comparative genomics analysis displayed that the cytochrome p450 gene family is extremely expanded to 425 members in FAW, of which 283 genes are specific to FAW. Treatments of Chinese populations with twenty-three pesticides showed the variant patterns of transcriptome profiles, and several detoxification genes such as AOX, UGT and GST specially responded to the pesticides. These findings will be useful in developing effective strategies for management of FAW in China and other invaded areas.

KEYWORDS Spodoptera frugiperda, chromosome-level genome, population differentiation, cytochrome p450, pesticides

\section{INTRODUCTION}

The fall armyworm (FAW), Spodoptera frugiperda, is a devastating and highly migratory insect herbivore native to tropical and sub-tropical areas in America, and was first reported more than 100 years ago in the USA as a damaging pest to maize and other crops (Hinds and Dew, 1915). Currently, FAW invasion has been reported in more than 100 countries worldwide (Fig. S1). FAW larvae may attack more than 350 host plant species belonging to 76 plant families (Montezano et al., 2018), causing serious damage to crops including maize, rice, sugarcane, sorghum, and cotton (Sparks, 1979). The strong migration capacity of FAW adults has led to its recently rapid and wide spread throughout

Furong Gui, Tianming Lan, Yue Zhao, and Wei Guo have contributed equally to this work.

Electronic supplementary material The online version of this article (https://doi.org/10.1007/s13238-020-00795-7) contains supplementary material, which is available to authorized users.
Africa, the Middle East, and several Asian countries including India, Bangladesh, Sri Lanka, Thailand, Yemen, Myanmar and China since their first appearance in west Africa in early 2016 (Goergen et al., 2016; Mallapur et al., 2018; CABI, 2019; Farmer, 2019). In January 2019, FAW was first reported in Yunnan Province of China (Jing et al., 2019), and then rapidly spread to 13 provinces and cities (Yunnan, Guizhou, Sichuan, Chongqing, Guangxi, Guangdong, Hainan, Hunan, Hubei, Fujian, Zhejiang, Jiangxi and Henan) of China within only four months (Wu et al., 2019). With the fast dispersal rates and available suitable habitats in the southwest, central and northern regions of China, the pest had already attacked 1,366 counties (cities and districts) across 24 provinces of China until August 17, 2019 (Xiao et al., 2020). The recent FAW invasion in China has caused tremendous losses of agricultural production in the invaded regions and has become a great threat to the corn production in eastern China, the major regions of corn production in China (Li et al., 2019).

The FAW consists of two morphological identical but genetically distinct strains, the corn strain ( $C$ strain) and rice strain (R strain) (Pashley, 1986; Juárez et al., 2014; Nagoshi et al., 2018). The C strain is the major type that widely spreads around the world. Each strain shows specific physiological traits thereby exhibiting strain-specific response to biological and chemical agents (Nagoshi and Meagher, 2004; Meagher and Nagoshi, 2012; Unbehend et al., 2014). The $\mathrm{C}$ strain larvae are more tolerant to pesticides methyl parathion, cypermethrin and Bacillus thuringiensis $(B t)$ d-endotoxins than the R strain (Adamczyk et al., 1997). Therefore, the confirmation of FAW strain type (s) invading China is critical for the effective management of the pest.

FAW management would benefit from detailed and comprehensive genomic analysis. However, the FAW genomes from its cell lines or strains are fragmented without anchoring on chromosomes before the year 2019 (Gouin et al., 2017; Kakumani et al., 2014; Nam et al., 2018, 2019; Nandakumar et al., 2017). With the advancement in genome sequencing and assembly technology, three chromosome-level genome assemblies of FAW have been published or pre-printed (Nam et al., 2019; Zhang et al., 2019, 2020; Xiao et al., 2020). Nevertheless, only two of them are available to the public (Xiao et al., 2020; Zhang et al., 2020). In addition, the genome sizes of the three assemblies varied dramatically from 384 to $486 \mathrm{Mb}$, even considering the sequencing technologies and potential strain differences. The quality of the genome assemblies is the foundation of population genetic analysis; therefore, more accurate FAW genome assembly and population genomes are urgently needed.

In this study, we comprehensively analyzed the results from de novo genome sequences, re-sequencing data of America, Africa, and China populations, as well as transcriptomic profiles after pesticide treatments. We assembled a chromosome-level genome using one male FAW collected from Yunnan, the first place of the pest occurred in China. 
We also analyzed the strain types of the FAW population that invaded Yunnan, and also discussed the possible origin of the invaded FAW populations in China. In addition, we screened expanded gene families in FAW to determine key genes associated with the function of polyphagia and resistance to pesticides. The results provide essential information for managing FAW invaded in China.

\section{RESULTS}

Genome assembly, evaluation and annotation

A total of $110.93 \mathrm{~Gb}$ high-quality single tube long fragment read (stLFR) sequencing data was generated from a male individual from Yunnan Province (Table S1 and Fig. 1A). The size of the primary genome assembled by the stLFR data is $542.42 \mathrm{Mb}$, and the scaffold N50 is $507.12 \mathrm{~kb}$. Using the High-throughput chromosome conformation capture $(\mathrm{Hi}-\mathrm{C})$ technology, the scaffolds were finally concatenated to 31 chromosomes, whereby scaffold N50 is $14.16 \mathrm{Mb}$. Genomes size assembled in this study fell within the scope of $246 \mathrm{Mb}$ to $809 \mathrm{Mb}$ of the Lepidopteran species (Triant et al., 2018). However, the size of the genome reported in the current study is larger than that of the previously assembled FAW genomes (Kakumani et al., 2014; Gouin et al., 2017; Nam et al., 2018, 2019; Nandakumar et al., 2017; Zhang et al., 2019, 2020; Xiao et al., 2020) (Table 1). The extra regions of
A

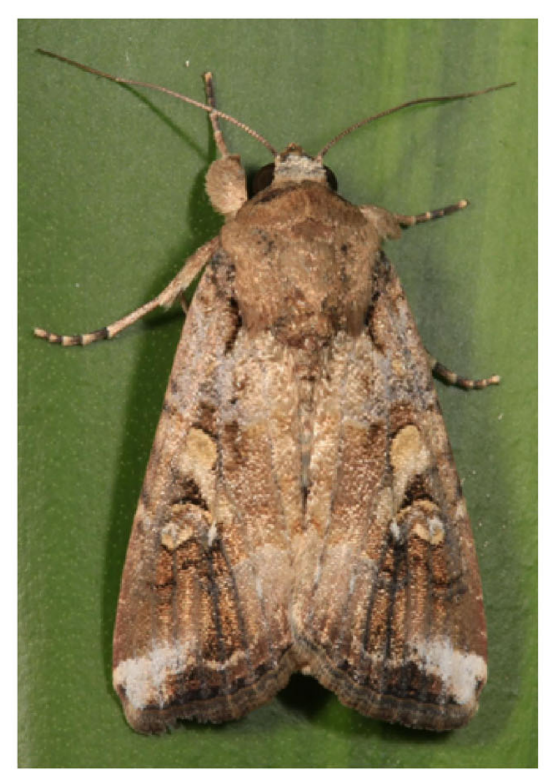

chr1 B
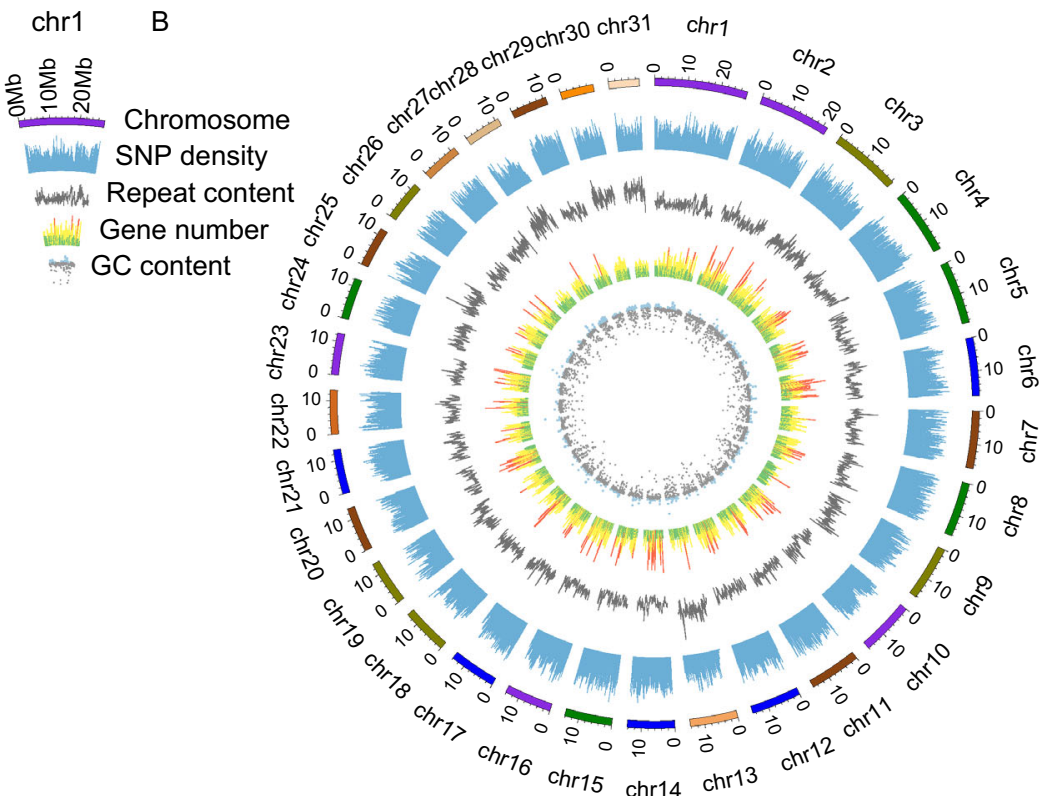

C

Spodoptera litura

19

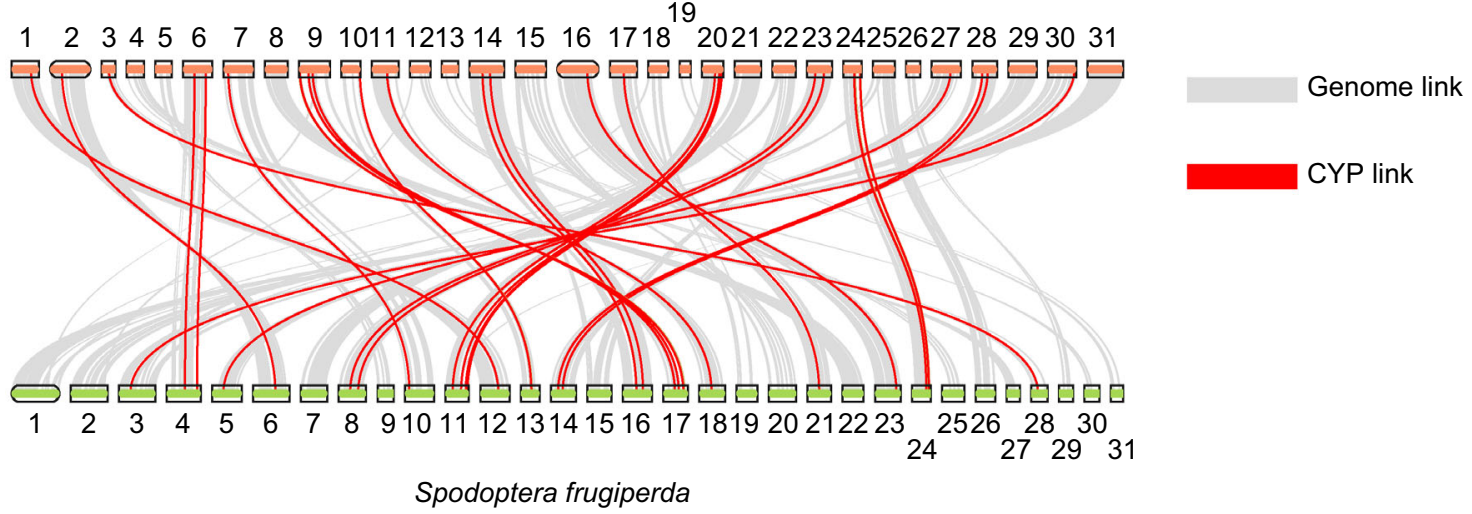

Figure 1. FAW genomic characterization and synteny with Spodoptera litura. (A) The male adult of fall armyworm (FAW) collected in Yunnan used for genome assembly. (B) Genomic characterization of FAW genome, it shows the GC content, gene number, repeat content, Single Nucleotide Polymorphism (SNP) density and chromosome from the center to the edge. (C) Synteny between FAW and Spodoptera litura genome. The gray lines reveal the genome-wide collinearity, and the red lines reveal the Cytochrome P450 (CYP) gene collinearity. 
Table 1. Comparison of SFynMstLFR and other published Spodoptera frugiperda genome assemblies.

\begin{tabular}{llllll}
\hline Assembly & ASM221328v1 & SF_CORN_1 & Sf-RVN WGS & ASM75363v2 & SFynMstLFR \\
\hline Bioproject & PRJNA380964 & PRJEB13110 & PRJNA344686 & PRJNA257248 & - \\
Assembly approach & Canu & - & CLC NGS Cell & SOAPdenovo & supernovo \\
Sequencing platform & PacBio & - & Illumina-HiSeq 2500 & Illumina/454/SOLiD & MGISEQ-2000 \\
Number of contigs & 2,844 & 50,014 & 89,958 & 119,777 & 29,584 \\
Contig N50 (bp) & 516,067 & 21,390 & 10,170 & 6,276 & 31,970 \\
Number of scaffolds & 2,396 & 41,562 & 66,318 & 37,235 & $31+21809$ \\
Scaffold N50 (bp) & 601,127 & 52,317 & 12,379 & 53,779 & $14,162,803$ \\
Total gap length (bp) & 890,870 & $11,379,136$ & $2,171,005$ & $27,684,486$ & $37,953,553$ \\
Total bases (bp) & $514,225,903$ & $436,269,483$ & $391,647,540$ & $358,047,567$ & $543,659,128$ \\
Ungapped bases (bp) & $513,335,033$ & $424,890,347$ & $389,476,535$ & $330,363,081$ & $505,705,575$ \\
\hline
\end{tabular}

our FAW genome compared with that published by Xiao (Xiao et al., 2020) are evenly distributed on 31 chromosomes (Fig. S2). We mapped the raw data (raw reads used for assembly of our genome and Xiao's genome) to those regions, and the mapping rates are more than $30 \%$, suggesting the validity of the extra regions. Besides, 1,494 genes were identified in the extra regions, of which 1,421 genes were expressed gene (average FPKM value of control $>0$ ) (Fig. S3) and 512 genes were differential expression genes (DEGs) in 23 pesticide treatments (Fig. S4), indicating the assembled extra regions might be genuine and functional.

The GC content of FAW genome is $36.52 \%$ (Fig. 1B) which fell within the scope of $31.6 \%$ to $37.7 \%$ of its closely related Lepidopteran species, Bicyclus anynana (Nowell et al., 2017). The genome covers $95 \%$ for complete BUSCO genes with duplicated BUSCO components of $9.8 \%$ (Table S2). The quality of these data was largely improved, because of more information available from other published FAW genomes (PRJNA380964, PRJNA257248, PRJEB 13110, and PRJNA344686) (Table 1), including the recently published FAW assembled using the Pacbio RSII technology (Xiao et al., 2020). The sequencing data generated from libraries for Hi-C, WGS, RNA-seq were also mapped to the assembled genome, and all the mapping rates and sequencing coverage were above $90 \%$ (Tables S3 and S4), indicated that most of the sequencing data can be mapped back to the genome, and the K-mer analysis showed that the inferred genome size were various, indicating the possibly big differences on genome size between different individuals (Fig. S5 and Table S5). Moreover, the FAW expressed sequence tags (EST) and transcripts from NCBI were perfectly mapped to the genome with $>90 \%$ and $>80 \%$ mapping ratios, respectively (Table S6).

Combining de novo and homology-based search, we identified $153 \mathrm{Mb}$ repeat elements, accounting for $28.24 \%$ of the FAW genome (Fig. 1B). A total of 22,201 genes were annotated using the repeat-masked genome based on de novo, RNA-seq and homology-based methods (Tables S7 and S8). The gene set covered $94.2 \%$ of complete BUSCO genes, which presented a more complete gene set than previously published FAW genome (Table S9). Totally, $93.48 \%$ of the identified genes in the gene set were identified as functional genes (Table S8). The FAW genome established in this study revealed a high sequence coverage and identity with its closely related species, Spodoptera litura (Fig. 1C), another important insect pest on vegetable and horticultural plants.

Comparative genomics revealed the possible genetic basis of pesticides resistance

Based on the 4,056,205 bp fourfold degenerate synonymous site (4DTv) sequences across the genomes of FAW and other seven Lepidopteran species, the phylogenetic analysis showed that FAW is closely related with S. litura (Fig. 2). Branch-site likelihood ratio test was performed to identify positively selected genes (PSGs) based on 20,051 singlecopy orthologous genes of FAW and Bombyx mori abstracted by syntenic alignment, and a total of 363 PSGs were identified, which were enriched in reverse transcriptase domain (IPR000477), endonuclease/exonuclease/phosphatase (IPR005135), zinc finger, CCHC-type (IPR001878), fructose-2,6-bisphosphatase (IPR003094), etc. (Table S10). Of them, ABCC4, UGT and ChuaMOX genes were related to detoxification function.

Several insect gene families are supposed to be associated with detoxification function, including ATP-binding cassette $(A B C)$ (Koenig et al., 2015), Carboxyl esterase (CES) (Satoh and Hosokawa, 2009), Cytochrome P450 (CYP) (Zhu et al., 2013), Glutathione S-transferase (GST) (Hayes and Pulford, 2008), UDP-glucuronosyl transferase (UGT) (Bock, 2016), Aldehyde Oxidase (AOX) (Chang et al., 2010), Chitinase Acidic (CHIA) (Downing et al., 2000), Epoxide Hydrolase (EPHX) (larmarcovai et al., 2008), PIF1 5'-to-3' DNA helicase (PIF) (Erlandson 2009), Patched Domain 


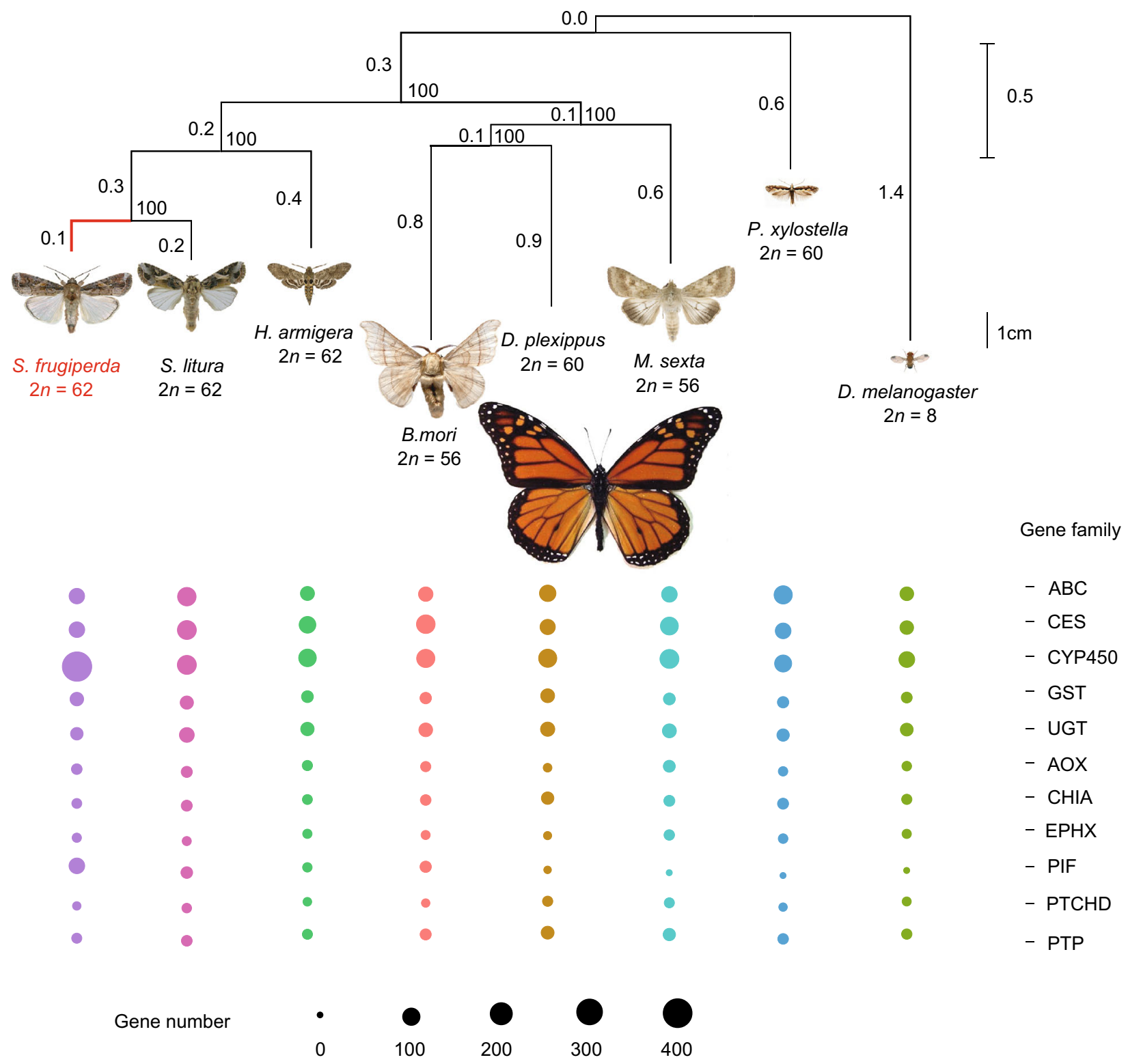

Figure 2. Phylogenetic relationships among seven lepidopteran and D. melanogaster genomes. Below: the gene family number of ATP-binding cassette ( $A B C$ ), Carboxylesterase (CES), Cytochrome P450 (CYP), Glutathione S-transferase (GST), UDPglucuronosyl transferase (UGT), Aldehyde Oxidase $(A O X)$, Chitinase Acidic $(C H I A)$, Epoxide Hydrolase (EPHX), PIF1 5'-to-3' DNA helicase (PIF), Patched Domain Containing $(P T C H D)$ and protein tyrosine phosphatase (PTP) genes.

Containing $(P T C H D)$ and Protein Tyrosine Phosphatase (PTP) (Herraiz et al., 2006) gene families. Here, we scanned all of these genes or gene families to detect expanded detoxification-related genes (Fig. 2 and Table S11). A total of 1,256 CYP genes were detected from these eight insect species, and 425 CYP genes were identified in the FAW genome, indicating that they are extremely expanded in FAW than the other seven Lepidopteran species. The significant expansion trend is similar with the published FAW genome (Xiao et al., 2020). Besides, the expanded CYP genes from FAW shows quite short-time divergence, and 283 genes are specific to FAW (Fig. 3A, 3C and Table S12). 


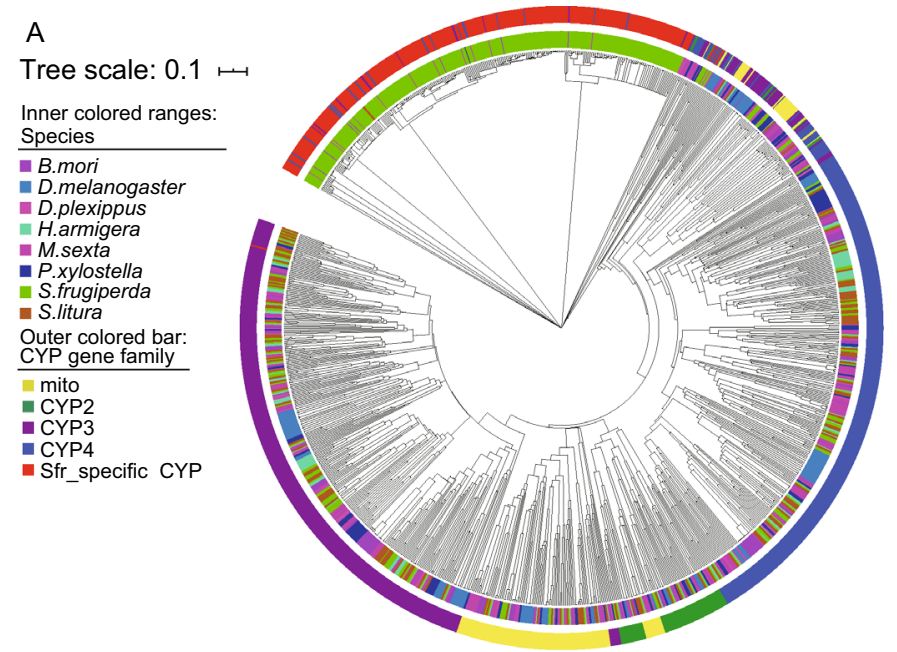

B

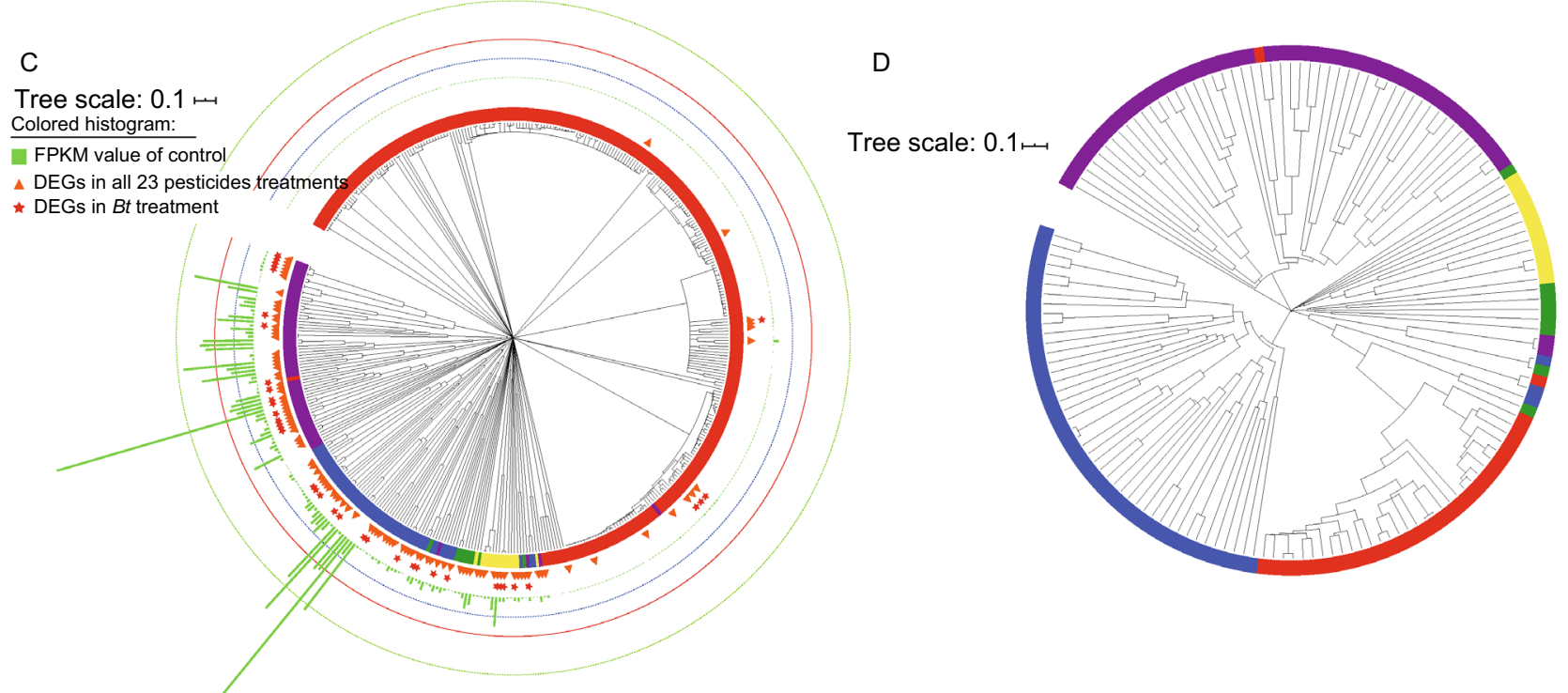

Figure 3. The gene tree of Cytochrome P450 gene family. (A) 1,256 Cytochrome P450 (CYP) genes in 8 species, the label color shows different species, the bar color shows different CYP gene sub-families. (B) The seq-logo plot of the motif and 10 bp flanking sequences of CYP genes in 3 species. (C) The 425 CYP genes of FAW, the bar color shows different CYP gene sub-families, 107 differential expression genes (DGEs) between all treatments and control are shown as orange triangle, 37 DGEs between $B t$ treatment and control are shown as red star, and the histogram means CYP gene FPKM value of control. (D) The 152 CYP genes filtered by CD-HIT, the bar color shows different CYP gene sub-families.

According to the DNA sequence identity, the 425 CYP genes of FAW were merged into 152 gene clusters (Fig. 3D).

Furthermore, the MEME software verified the accuracy of the CYP gene family for the FAW-specific CYP gene set based on the control CYP genes from $B$. mori and $S$. litura. The conservation of motif and $10 \mathrm{bp}$ flanking sequences is shown as a seq-logo plot (Fig. 3B). From the highlighted seqlogo plot, the conservation of motifs in the three species (FAW, B. mori, and S. litura) displayed a striking variant. The motif sequence in FAW is more conserved than in the other two species.

\section{Whole genome resequencing}

\section{Variation discovery}

To assess the genetic diversity among FAW accessions, 163 individuals from America $(n=39)$, Africa $(n=24)$ and China $(n=100)$ (Table S20) were mapped to the FAW assembly (Fig. 4A). The average mapping rate and sequencing coverage were $95.60 \% \pm 3.34 \%$ and $90.41 \% \pm 2.38 \%$, respectively (Table S4). After filtration (See "METHODS"), we identified 45,569,102 single nucleotide polymorphism (SNPs) and 11,391,362 short genomic insertions and 
deletions (InDels) (less than $40 \mathrm{bp}$ ) with a minor allele frequency (MAF) $>0.01$. About $71.71 \%$ of the SNPs are located in the intergenic regions, whereas $3.99 \%$ of them are in the coding sequences. The non-synonymous-to-synonymous substitution ratio for the SNPs in the coding regions is 0.242 , indicating high quality of the SNP call sets. Moreover, $72.94 \%$ of the InDels are located in the intergenic regions, whereas $0.38 \%$ of them in the coding regions. About $68.77 \%$ of the InDels in the coding regions were estimated to cause frame shift mutations. The SNP density is shown in Figs. 1B and S6.

\section{African origin of FAW populations in China}

The core set of SNPs were used to analyze the phylogeny and population structure of FAW. Principal component analysis (PCA) showed substantial genetic diversity among the six FAW populations, $\mathrm{PC} 1, \mathrm{PC} 2$ and $\mathrm{PC} 3$ revealing a total genetic variance of $5.49 \%, 5.34 \%$ and $2.70 \%$, respectively (Fig. 4B). PC1 separated American FAW from other five accessions, PC2 evidently separated the four American accessions and PC3 separated AA accessions from the rest. American populations showed relatively large genetic divergence within populations, compared to Chinese and African populations.

Maximum likelihood (ML) phylogenetic analysis revealed distinct monophyletic clades for Ethiopia (ET), Kenya (KE), America (A), South Africa (SA), Guangxi (GX), and Yunnan (YN) FAW populations (Fig. 4C). This ML-tree further illustrated that the American group could be marginally divided into subgroups $A A$ and $A B-A C-A D$ (Fig. 4C). Chinese (YN, $\mathrm{GX}$ ) and African (ET, KE, SA) clades displayed close phylogenetic relationship (Fig. 4C). Structure analysis showed that populations in China and Africa comprise accordant components (Fig. 4D and 4E, $K=5$ ). Varying the number of $K$ (presumed ancestral populations) revealed genetic distinctions between American and other accessions. When $K=2$, we observed two separate clusters: American $(A)$ and other accessions, and when $K=3$, three separate clusters were exhibited: $A A, A B-A C-A D$ and other accessions. Similar patterns are also supported by the PCA and ML-tree analyses (Fig. 4B and 4C), suggesting that Chinese accessions ( $Y N$ and $G X$ ) shared much closer genetic relationships with African accessions (SA, ET, KE) than that of America (AA, AB, AC, AD). Taken together, FAW populations in China (YN, GX) migrated directly from Africa, because the genetic structure of these populations is highly mixed. Besides, we estimated admixture graphs of different geographically defined groups using TreeMix. Extensive gene flow $(45.17 \%)$ were found from $A B$ to $A C$ when $m=1$ (Fig. S7). Additional gene flow from KE to ET (14.66\%) and YN $(15.17 \%)$ were also found when setting the parameter $\mathrm{m}=2$ and $m=3$, respectively. These findings proved that American accessions are deep divergent from other accessions, further supporting that Chinese accessions was derived from Africa, rather than directly from America.
All the samples were identified as C strain in FAW populations invading China

In our study, we identified 240 samples by using TpiE4-183, including 163 resequencing samples, 72 RNA-seq samples used for pesticide resistance analysis, one sample used for genome assembly survey, one sample used for stLFR genome assembly, one sample used for $\mathrm{Hi}-\mathrm{C}$ sequencing and two RNA-seq samples used for gene annotation (Tables S1 and S20). Interestingly, all 100 samples from China and 24 samples from Africa were identified as $C$ strain, while both $C$ strain and $R$ strain were found in the American samples (Fig. S8). At present, only $C$ strain was found in the Chinese and African populations, indicating all these samples have similar genomic backgrounds.

\section{Differentiation of FAW demographic histories}

To reveal the demographic and geophylogenic history of FAW, a pairwise sequentially Markovian coalescent (PSMC) model was used to analyze SNP data from FAW populations of America ( $n=10$, three from AA, three from AB, two from $A C$, and two from AD), Africa ( $n=10$, four from $E T$, four from $\mathrm{KE}$ and two from SA) and China ( $n=10$, five from $\mathrm{YN}$ and five from $G X$ ). The results were scaled to the real time by assuming a generation time of 0.25 year and a neutral mutation rate of $2.9 \times 10^{-9}$ per year (See "METHODS"). From the one Mya (million years ago), the effective population size of each subgroup was found to be less than 800,000 (Fig. S9). Subsequently, African populations (except for $\mathrm{KE}$ ), all Chinese populations and $A B$ population from America exhibited a mild $\mathrm{Ne}$ expansion in 100-400 Kya (thousand years ago) ( $\mathrm{Ne} \approx 500,000-1,000,000)$, which levels off during 10-100 Kya ( $\mathrm{Ne} \approx 750,000-1,000,000$ ). However, American accessions (except for $A B$ ), as well as the KE population from Africa, exhibited a rapid Ne expansion during 9-200 Kya ( $\mathrm{Ne}$ from 500,000 to even $16,000,000)$. Subsequently in $100 \mathrm{Kya}$, Chinese accessions, as well as ET, SA and $A B$ populations had a rapid Ne contraction $(\mathrm{Ne} \approx 100,000)$. At 7-10 Kya, American accessions $(\mathrm{AA}, \mathrm{AC}$ and $\mathrm{AD})$ had a rapid $\mathrm{Ne}$ contraction as well $(\mathrm{Ne} \approx$ $50,000)$. Overall, we found extremely similar demographic history within Chinese FAW populations, which displayed more similar demographic distribution patterns with most African populations than with American populations.

\section{Genetic diversity, population differentiation and natural selections}

Across the FAW genome, the global nucleotide diversity $(\pi)$ is well correlated to the global SNP and InDel density (Fig. S6). Subsequent analysis revealed that America accessions were highly polymorphic with $A B$ $\left(\pi=1.02 \times 10^{-3}\right)$ being less polymorphic than $\mathrm{AA}$ $\left(\pi=2.59 \times 10^{-3}\right)$. AC had a nucleotide diversity of $1.95 \times 10^{-3}$, whereas that of $A D$ was $1.74 \times 10^{-3}$. KE 


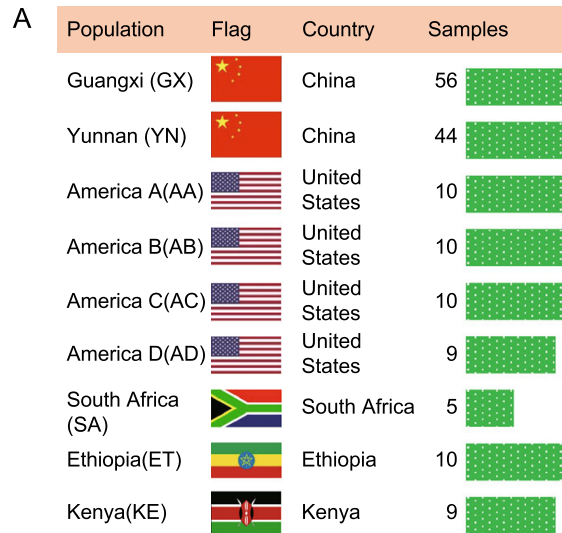

B
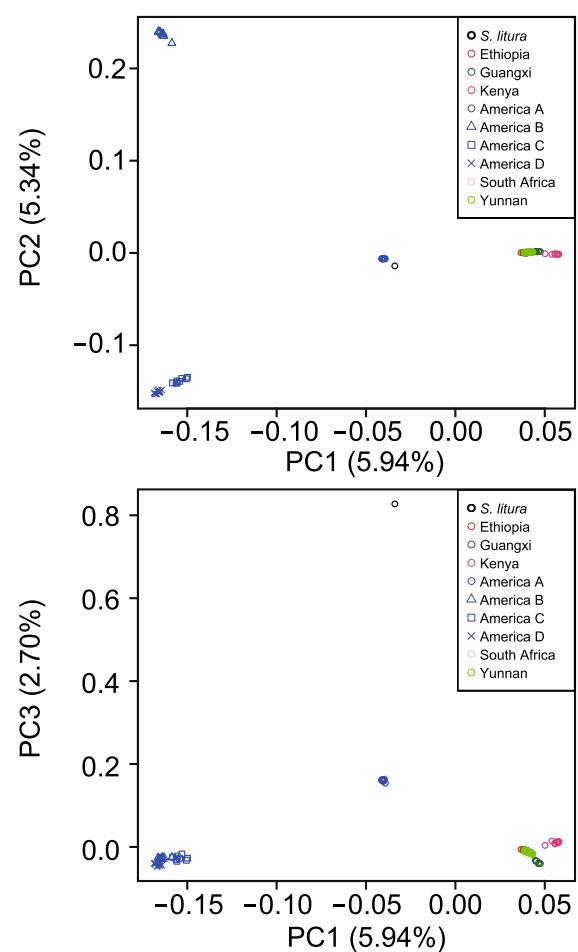

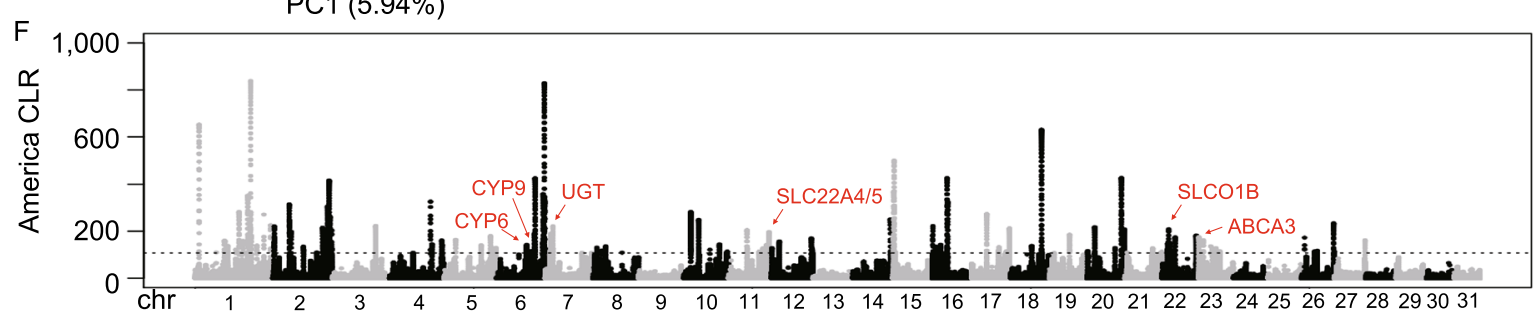

E

$K=2$

$K=3$

$K=5$

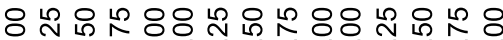

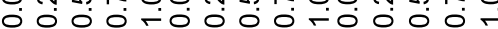

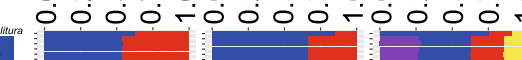

D
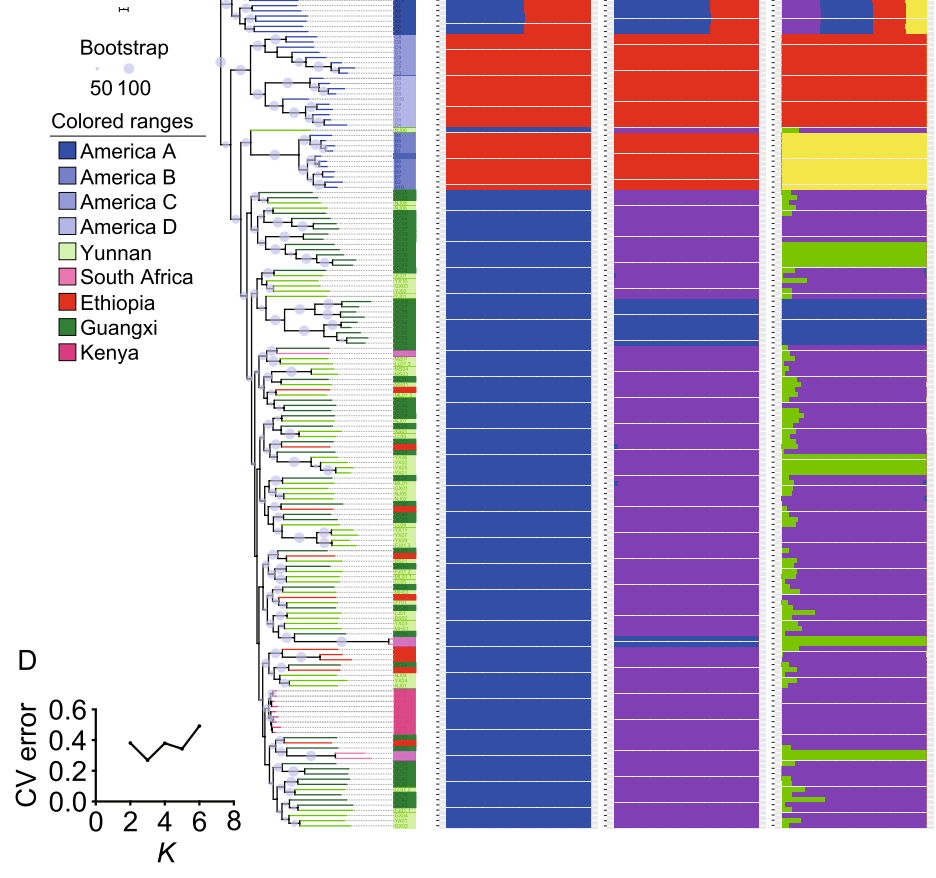

Colored range

America A

$\square$ America C

$\square$ America D

$\square$ Yunnan

$\square$ South Africa

$\square$ Ethiopia

Guang
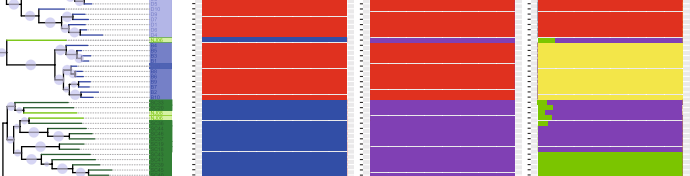

\section{$=$}
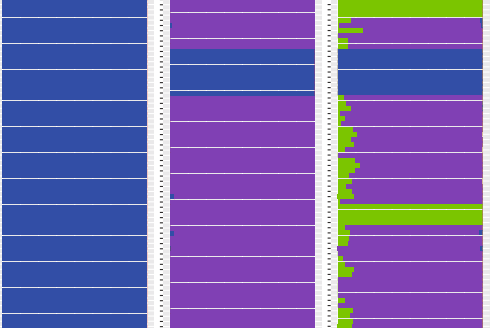

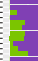

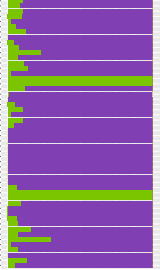

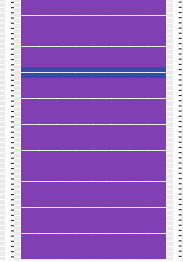

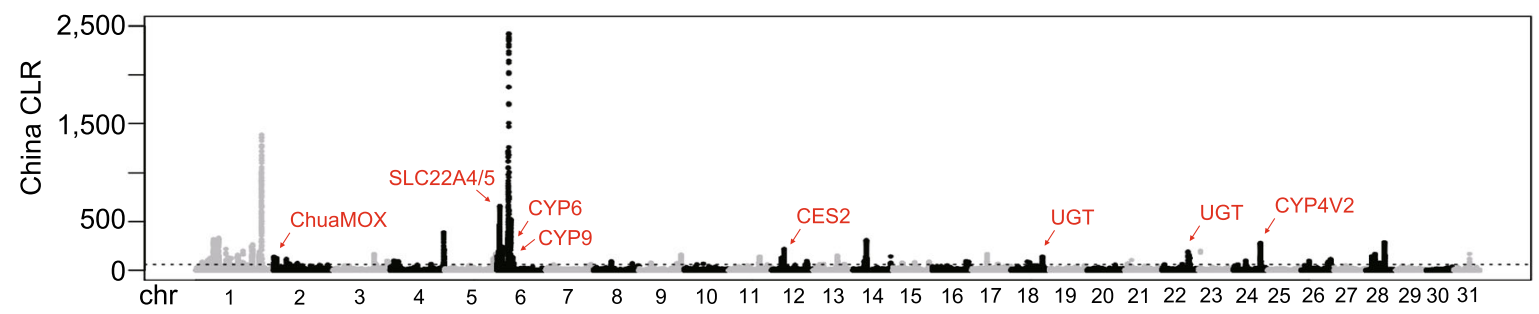


Figure 4. Phylogeny and population structure of FAW. (A) Sampling locations of six geographical populations. (Ethiopia: red, Kenya: light red, America: blue, South Africa: pink, Guangxi: green and Yunnan: light green) (B) PCA plots of the first three components of major FAW accessions using whole-genome SNP data. (C) Maximum likelihood phylogenetic tree of FAW population (from what data). Fabricius (Spodoptera litura) was used as an outgroup. (D) Cross-validation (CV) error of ADMIXTURE. (E) Population structure of major FAW categories estimated by ADMIXTURE. Each color represents one ancestral population. Each accession is represented by a bar, and the length of each colored segment in the bar represents the proportion contributed by that ancestral population. (F) CLR scores calculated by SweeD across the genome in both American D and Yunnan accessions. The dashed lines mark the regions at the top $0.5 \%$. The red arrow indicates the detoxification candidate gene regions.

accession exhibited the highest nucleotide diversity of $3.51 \times 10^{-3}$ (Fig. S10 and Table S13).

$F_{\text {ST }}$ (Weir and Cockerham's estimator) analysis indicated that $A B$ branch had the greatest genetic divergence compared with the other eight accessions (Table S14), consistent with the result of nucleotide diversity analysis (Fig. S10 and Table S13). AD branch displayed great genetic divergence among SA, ET, KE, YN and GX accessions (Table S14) compared to American accessions. Thus, Chinese FAW populations were evolutionally closer to African FAW than American FAW, further suggesting that Chinese FAW originated from Africa.

We also performed to investigate potential selective signals in the genomes of each FAW group by identifying regions (phromosome length/1000) that scored top $0.5 \%$ in the model. The selective sweep signals and harbored gene number are shown in Table S15. As the different management strategies between America and China, which Bt crop has been used in America for a long history, whereas chemical pesticides have been largely applied in China, American accession ( $A A, A B, A C$ and $A D)$ and Chinese accession (YN and $G X$ ) were selected to detect the differences. In American accession, CYP6, CYP9, UGT, $S L C 22 A 4 / 5, S L C O 1 B$ and ABCA3 genes were under positive selection. In Yunnan accession, ChuaMOX, SLC22A4/5, CYP6, CYP9, CES2, UGT and CYP4V2 genes were detected to have signals of selective sweep (Fig. 4F).

\section{Transcriptomic responses of FAW to pesticides exposure}

To investigate the transcriptomic profiles of FAW to pesticides, twenty-three pesticides including four biological, ten single and nine mixed chemical pesticides were applied to the third-instar larvae of FAW (Fig. 5 and Table S16). The FAW displayed differential responses to different pesticides with regard to the transcriptional expressions (Fig. 5A). Among the biological pesticides, Bacillus thuringiensis (P2) induced the most dramatically transcriptional expression with 1,231 up-regulated and 824 down-regulated genes. In the single and mixed chemical pesticides, eleven pesticides treatments induced the differential expressions of more than 1,500 genes. Then we obtained a union set with 7,991 differential expression genes (DEGs) by merging the DEGs after 23 pesticides treatment. Further clustering of the 7,991 DEGs categorized the 23 pesticides-treated expression profiles into four clusters (Fig. 5B). The four pesticide clusters represent differentially functional groups that effective (Clusters 3 and 4) or ineffective (Clusters 1 and 2) to FAW pest control. Several most effective chemical pesticides in FAW management, such as emamectin benzoate, spinetoram, chlorantraniliprole and cyantraniliprole are concentrated in Clusters 3 and 4, irrespective of single or mixing with other chemicals. Ryanodine receptor-targeted pesticides, such as flutolanil (P18), thiamethoxam (P19), cyantraniliprole (P21) and chlorantraniliprole (P23) were clustered together in Cluster 4 . Emamectin benzoate-containing mixture pesticides were clustered with ryanodine receptor-targeted pesticides in Cluster 4. Different with other two biological pesticides, Bacillus thuringiensis and azadirachtin (P2 and P4) were also clustered in Cluster 4 (Fig. 5B).

The 107 differentially expressed $P 450$ genes were further clustered (Fig. 5C). Cluster I represented 48 up-regulated genes, including 2 CYP2, 26 CYP3, 5 CYP4, 6 mitochondrial CYPs and 9 FAW-specific CYPs after 23 pesticides treatment (Fig. 5C). Cluster II represented 22 down-regulated genes, including 3 CYP3, 13 CYP4, 2 mitochondrial CYPs and 4 FAW-specific CYPs after 23 pesticides treatment (Fig. 5C). Further KEGG enrichment analysis showed that cutin, suberine and wax biosynthesis (map00073) pathways were mutually up-regulated after nineteen pesticides exposure (Table S17). Meanwhile, many sugar metabolism pathways, such as fructose and mannose metabolism (map00051), amino sugar and nucleotide sugar metabolism (map00520), galactose metabolism (map00052) and pentose and glucuronate interconversions (map00040) pathways were mutually down-regulated after variant pesticides treatment (Table S17).

In addition, we focused on some detoxification gene families such as CYP, UGT, GST, ABC and CES to identify the potential pesticides resistance genes:

\section{CYP gene}

Under the selection of pesticide, many insects have evolved to produce new CYPs, or CYP gene family expands, like Plutella xylostella (You et al., 2013), Aphis glycines (Wenger et al., 2017) and Bemisia tabaci (Chen et al., 2016) and so on. New CYP genes or largely expanded CYP gene family 
A

Number of genes

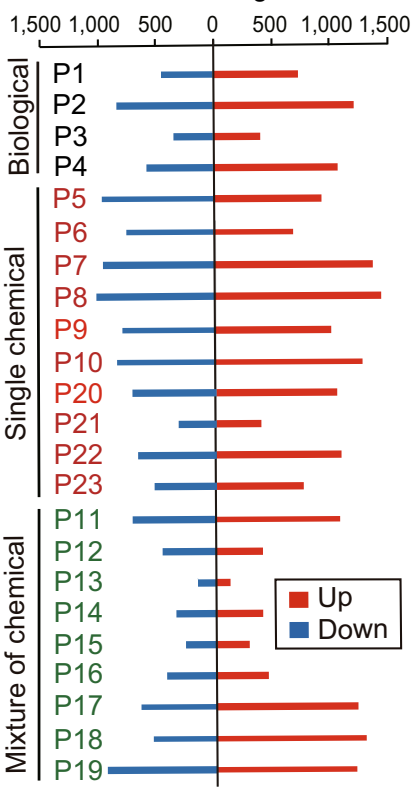

B

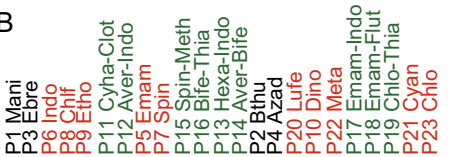

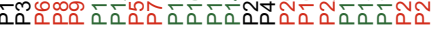

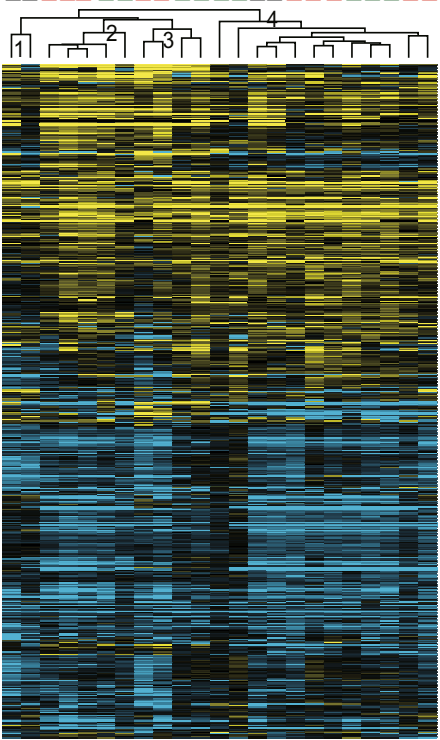

C

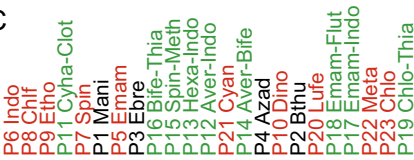

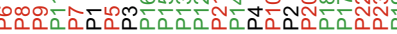

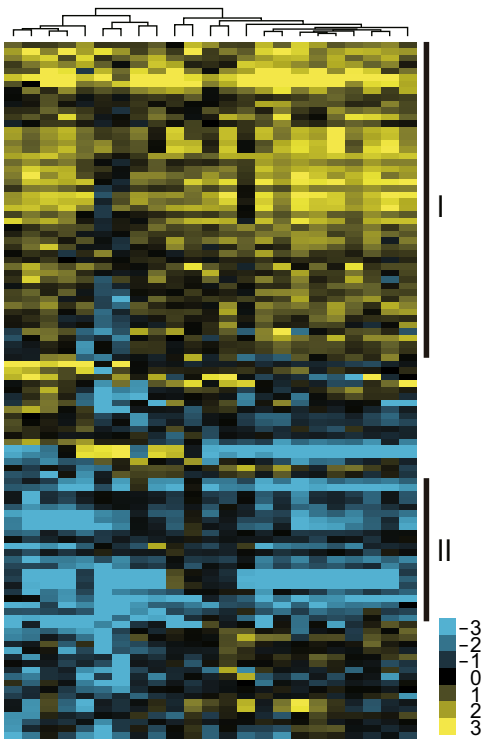

D

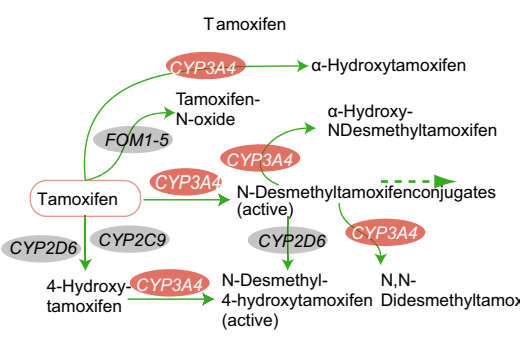

Cyclophosphamide \& Ifosfamide

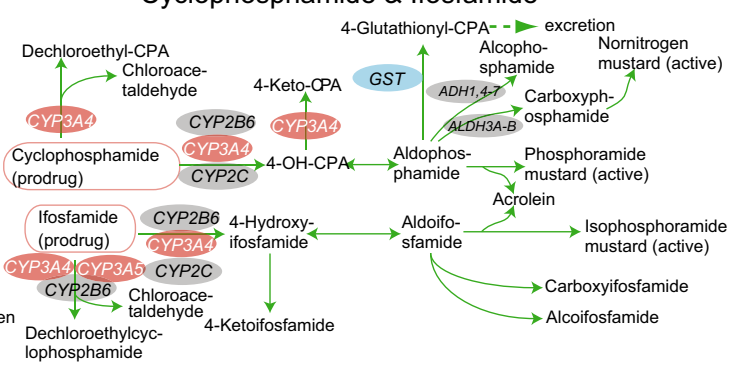

Dechloroethylcyc-
lophosphamide

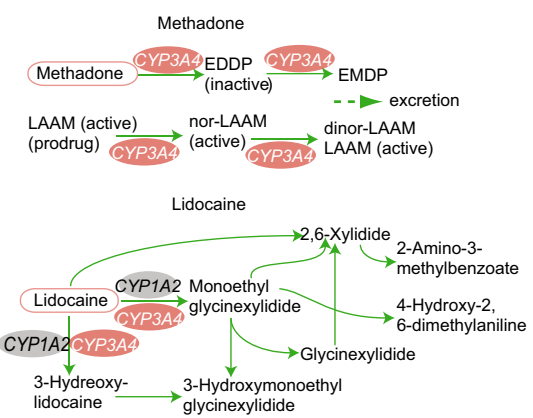

lidocaine

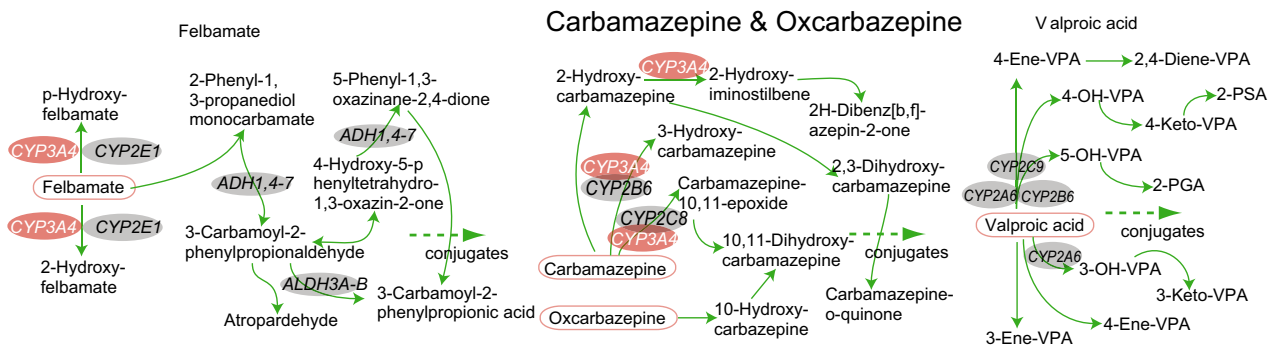

Codeine \& Morphine

Citalopram-N-oxide

CYP2D6

Citalopram

CYP2C19 MAOA-B

CYP2D 6 CYP3A4 $-\begin{aligned} & --- \\ & \text { conjugates }\end{aligned}$

N-Demethyl-MAOA-BCitaloprama-

Citalopram $\longrightarrow$ MAOA-BCitalopra

CYP2D6 MAOA-BAOX1

$\begin{array}{ll}\mathrm{N} \text {-Demethyl- } & \text { Citalopram- } \\ \text { Citalopram } & \text { propionic acid }\end{array}$ $\rightarrow$ Codeine-6-

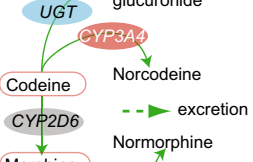

Morphine Normorphin

UGT

UGT Morphine-3-glucuronid

Morphine-6-glucuronid

(active)

Carbamazepine \& Oxcarbazepine _ valproic acid 
Figure 5. Gene expression profiling after twenty-three pesticides exposure. (A) Number of differential expression genes (DEGs) after each pesticide treatment. (B) Pesticides clusters using the combined 7991 DEGs. (C) The heatmap of 107 DGEs of FAW cytochrome P450 genes. X axial shows the different treatment (P1-P23), the color shows the fold change of DGEs, yellow means up regulation, blue means down regulation, black means the gene is not DEG in one/some treatment (s). (D) DGEs of Bt involved in hsa00982 pathway. The red means up-regulated DEGs, the blue means down-regulated DEGs.

have potential roles in detoxification and/or pesticide resistance.

In this study, a remarkable change in the expression of the CYP genes was observed when FAW individuals were treated with the pesticides. Indeed, many CYP genes (107/ 425) were differently expressed as shown in Fig. 3C and 3D. Fold change values between treatments and controls in the 107 DEGs are shown in Table S18. There were 2,055 DEGs between $B t$-treated larvae and controls, which contained 37 CYP genes including CYP4, CYP6, CYP9, CYP12 and CYP49 (Figs. 3C and S11). CYP49 and most of CYP4 showed down regulation, while CYP6, CYP9 and CYP12 exhibited up regulation, which related to pesticide resistance even the cross resistance in multiple pesticides. Bt could be an effective pesticide target at many gene like CYP. Moreover, the expression of DEGs was significantly elevated in drug metabolism-cytochrome P450 (hsa00982, Fig. 5D) and metabolism of xenobiotics by cytochrome P450 (hsa00980).

\section{UGT gene}

Glucuronidation is a major conjugation reaction catalyzed by UGT family, which is associated with detoxification process reported in many insect genomes, such as $P$. xylostella (You et al., 2013) and B. tabaci (Chen et al., 2016). Transcriptome analysis indicated that all 49 UGT genes were detected as expressed genes (FPKM > 0 in any repetition) (Fig. S12), of which 22 genes were DEGs between 23 treatments and control. Notably, there were 9 down-regulated DEGs between chlorfenapyr treatment and control as well as 8 down-regulated DEGs between spinetoram water treatment and control. Glucuronic acid could combine with many harmful substances in insect liver, aiding in the detoxification process, indicating that the two types of pesticides may target UGT gene effectively.

\section{GST gene}

GST catalyzes the binding of nucleophilic glutathione to various electron-friendly exogenous chemicals, critical in toxicology. Isomers of GST display unusual broad-spectrum catalysis as well as the ability to sequester non-substrate drugs and hormones. Twenty-seven out of 29 GST genes were identified as expressed genes (Fig. S13), and 14 genes were DEGs between 23 treatments and control. There were 9 down-regulated DEGs between spinetoram water treatment and control, as well as 6 down-regulated DEGs between chlorfenapyr treatment and control. As a critical role of GST gene in toxicology, these two types of pesticides may target at GST gene effectively.

\section{$A B C$ gene}

Research shows that transporters, particularly subfamilies $B$ and $\mathrm{C}$, both of which are involved in detoxification and multidrug resistance, are up-regulated in the gut when larvae feed on plants (Koenig et al., 2015). Besides, ABC transporters are targets of Bacillus thuringiensis insecticidal toxins (Tay et al., 2015). In this study, 57 out of 58 ABC gene families ( $A B C A 3$ and $A B C C 4)$ were expressed genes (Fig. S14), and 21 genes were DEGs between 23 treatments and control. There were seven down-regulated DEGs between Emamectin*Flutolanil microemulsion treatment and control, implying the $A B C$ gene family may be a potential effective target for combination of Emamectin and Flutolanil.

\section{CES gene}

The CES genes encode members of the large carboxylesterase family, which are also involved in detoxification or metabolic activation of various drugs, pesticides, environmental toxins and carcinogens (Satoh and Hosokawa, 2009). In this study, all 30 CES genes (CES1 and CES2) were detected as expressed genes (Fig. S15), and of which, 16 genes were DEGs between 23 treatments and control. There were eight down-regulated DEGs between Metarhizium anisopliae treatment and control as well as between chlorantraniliprole treatment and control, indicating that the CES gene might be a potentially effective target for Metarhizium anisopliae and chlorantraniliprole.

\section{Other genes}

Apart from the above genes (families), there were other DEGs under varied treatments. SLC22A3, SLC22A4 and $S L C 22 A 5$ participated in poly-specific transportation of organic cations in the many organs, critical for elimination of many endogenous small organic cations as well as a wide array of drugs and environmental toxins. There were 51 expressed solute carrier family 22 (SLC22A) gene member (Fig. S16). Interestingly, there was at least one DEG between each treatment and control, and the total DEG number was thirty-one. There were 21 DEGs between spinetoram water treatment and control, and 16 DEGs between chlorantraniliprole*thiamethoxam treatment and control.

All 60 ChuaMOX genes were detected as expressed genes (Fig. S17), and 41 genes were DEGs between all 23 
treatments and control. Enzyme encoded by ChuaMOX is similar to one produced in sacs of millipede Chamberlinius hualienensis. In millipedes, the sacs rupture during defensive behavior, producing benzoyl cyanide-a protective chemical. There were over 15 ChuaMOX DEGs in seven treatments (one biological, four single and two mixed chemical pesticides). Besides, SULT1E1, NNT, PTPMT1 and $J H E H$ genes showed differential expression in different treatments.

Azadirachtin is a chemical compound in limonoid group in seeds as a secondary metabolite. There were 1,657 DEGs between azadirachtin treatment and control, which significantly enriched in detoxification (hsa04146), drug metabolism -cytochrome P450 (hsa00982, Fig. 5D) and metabolism of xenobiotics by cytochrome P450 (hsa00980) about 34 KEGG pathways (Table S19). There were 15 DEGs (Fig. S18) involved in hsa00982 and hsa00980, indicating that similar to $B t$ toxin, azadirachtin pesticide regulates expression of detox gene as well.

$B t$, Empedobacter brevis and azadirachtin act on the digestive system of insects. There were 199 DEGs (Fig. S19) overlapped among them, including PTPMT1 and JHEH, CES1, ChuaMOX, SLC22A4 and SLC22A5 genes associated with detoxification were also among the overlapped DEGs.

\section{DISCUSSION}

Taking the advantages of stLFR and $\mathrm{Hi}-\mathrm{C}$ technologies with long fragment reads, super high coverage and reads quality, we de novo assembled a high-quality FAW genome, which is so far the largest FAW genome assembly with a size of 542.42 Mb (Nandakumar et al., 2017; Zhang et al., 2019, 2020; Xiao et al., 2020). The genome of FAW was greatly different (Kakumani et al., 2014; Gouin et al., 2017; Nam et al., 2018, 2019; Nandakumar et al., 2017; Zhang et al., 2019, 2020; Xiao et al., 2020) (Table 1), which led to the different genome size and unmapped reads among different individuals. However, the FAW genome assembled in the current study is a high-quality genome in most of the key parameters, including the BUSCO evaluation, WGS mapping rate and RNA mapping rate. The quality improvement of the genome may facilitate further biological studies of FAW and help to develop effective strategies for management of this severely invasive pest worldwide. And the individual difference among FAW will be further studied by a pan-genome in the future.

In this study, we identified extremely expanded CYP gene family with 425 members (clustered into 152 clusters) compared with those in other assembled FAW genomes or closely related insect species. And the 169 CYP genes were identified in the latest research (Xiao et al., 2020), which is close with the CYP cluster number in this study. As the CYP gene family has the function of intensified detoxification (Cheng et al., 2017), the expansion of CYP gene may be associated with the highly polyphagous feeding behaviors of FAW, and its capability to inactivate or degrade xenobiotics to harmless chemicals. Moreover, FAW is frequently controlled by $B t$ crops in America, where the insect pest is originally from, and may have developed multiple resistance to not only pesticides, but biological agents and transgenic crops as well (Morillo and Notz, 2001; Santos-Amaya et al., 2016). The expansion event of CYP genes occurred in a short time, implying a rapid adaptation after the application of pesticides. On the other hand, the CYP genes annotated as known clan nearly all be DEGs, while the FAW-specific CYP genes can hardly be DEGs, which may due to the recent birth of these genes so there is no mature or complete function. However, the resistance caused by the expansion and evolution of CYP could hinder the control of FAW and may become the barrier against the usage of $B t$ crops over time. The CYP gene family might be valuable genetic targets in developing effective pesticides for FAW management. The results indicated that FAW is highly polyphagous and may be more pesticide-resistant than the other seven species in this study. The identification and mechanism of detoxification of FAW-specific CYP requires further investigation.

Resequencing genomic data of FAW from America presented a high genetic diversity, which was consistent with the American origin of FAW (Goergen et al., 2016; Mallapur et al., 2018; Jing et al., 2019). However, the genetic relationship between American populations and African and Chinese group is much far than that between African and Chinese populations. Instead, the extensive allele admixture between the FAW from Africa and China further support that FAW in China was highly likely from African populations, but not from America directly. Among four American populations, $A A, A B$ and $A C / A D$ displayed dramatic discrimination, which is probably partly due to strain differences, because we identified different predominantly strains in different populations (Fig. S8C). In addition, the identification of FAW subtypes in China using Tpi gene showed only the $\mathrm{C}$ strain, demonstrating an overwhelming proportion in the Chinese FAW population, which was in consistent with the result from Nagoshi et al. (2020). Tpi gene was frequently used for subtype identification, but with limitations therefore more markers should be developed in the future to make more accurate determination. The strain identification of the Chinese FAW populations is extremely important for making effective strategies to manage the pest in the country.

One of the main factors making pest control difficult is the evolution of pesticide resistance. Resistance in pest populations usually evolves within 2 to 20 years after application of new types of pesticides such as carbamates, organophosphates, pyrethroids, and Bt as a microbial or transgene pesticide (Daly et al., 1998; Weston et al., 2013). The study evaluated the differential expression of genes following exposure to 23 pesticides, which would provide theoretical evidence for appropriate selection and application of pesticides. Most pesticides exhibited similar KEGG pathway enrichments, particularly detoxification (hsa04146), drug metabolism-cytochrome P450 (hsa00982) and metabolism of xenobiotics by cytochrome P450 (hsa00980). This implies that different detoxification genes were up-regulated to resist variant 
pesticides in FAW. Nonetheless, we conclude that CYP plays a pivotal role in resisting pesticides. Thus, our findings on DEGs, particularly CYP genes, provide a strong understanding on general resistance to pest-control interventions.

In summary, we assembled a complete chromosomelevel genome of a male FAW from Yunnan Province, China. A total of 22,201 genes were predicted in this genome. Cytochrome P450 gene family in FAW was found extremely expanded, which was closely related to detoxification and tolerance to pesticides. Transcriptome analysis of 23 pesticide treatments revealed several candidate target genes. Besides, resequencing analysis of nine populations from six geographical locations in China, Africa and America demonstrated that the FAW in China was likely from Africa. Together, these findings are essential for developing effective strategies to manage the invasive FAW in China and other countries.

\section{METHODS}

\section{Samples and treatments}

A total of $240 \mathrm{FAW}$ samples were collected in this study, including 5 samples used for genome assembly, 72 samples used for RNA-seq analysis with 23 pesticide treatments and one control ( 3 biological sample duplicates (Table S16)), and 163 samples used for population resequencing. These samples collected from three continents, which were America $(n=39, \mathrm{~A})$; Africa $(n=24$; including South Africa $(n=5, \mathrm{SA})$, Ethiopia $(n=10, \mathrm{ET})$, Kenya $(n=9, \mathrm{KE}))$; and Asia (China, $n=177$; including Guangxi ( $n=56, \mathrm{GX})$, Yunnan $(n=118$, YN) and Guangdong $(n=3)$ ) (Table S20). American population was divided into four sub-populations: Population A was collected from grasses (braidleaf signalgrass, Urochloa platyphylla) in Franklin Parish, Louisiana on August 1st, 2019. Field-collected larvae were reared in a meridic diet (Ward's Stonefly Heliothis diet, Rochester, $\mathrm{NY}$ ) in the lab until adult emergence. Population B was collected from rice in a greenhouse in East Baton Rouge Parish, Louisiana in 2017, and has been reared in the meridic diet mentioned above since it was collected from the greenhouse. This population survived well on rice plants in greenhouse trials. Population $\mathrm{C}$ was collected from corn field in Franklin Parish, Louisiana in 2016. It has been reared in the meridic diet mentioned above since it was collected from the field, and survived well on corn plants. Population D was a hybrid of three populations, which were collected from corn plants in Collier County, Florida in 2011, Tift County, Georgia in 2013 and Franklin Parish, Louisiana in 2016, respectively. These field-collected larvae have been reared in the meridic diet mentioned above since they were collected from the field, and also survived well on corn plants.

All samples were subjected to intestinal and ovarian cleaning before DNA and RNA extraction.

DNA extraction, library preparation, sequencing, and genome assembly and annotation

High molecular weight DNA was extracted using the separated muscle tissue following the protocol recommended by Wang et al
(2019). A single tube long fragment read (stLFR) technology (Wang et al., 2019) was used to prepare the co-barcoding DNA libraries using the MGIEasy stLFR Library Prep Kit (lot number: 1000005622). The libraries were sequenced following the MGISEQ2000 protocol (Huang et al., 2017). A DNA library with a $5 \mathrm{~kb}$ insert size was constructed to verify the accuracy of genome assembly and sequenced by BGISEQ-500 sequencer. To finally ligate the scaffolds to chromosomes, Hi-C technology (Lieberman-Aiden et al., 2009) was used to capture the chromosome conformations using one adult FAW. The Hi-C libraries were prepared using the muscle tissues, as described previously in Lieberman-Aiden et al. (2009). Briefly, 1) Chromatin was cross-linked in the intact nucleus with formaldehyde; 2) Restriction endonuclease Hind III was then used for digestion to generate the cohesive terminus; 3) Filling ends of DNA fragments while marking with biotin; 4) Ligation of the neighboring DNA fragments; 5) Purifying and shearing the DNA fragments and performed the biotin pull down and adaptor ligation. The libraries were subjected to BGISEQ-500 sequencer for pair-end sequencing. The Hi-C library provided $167 \times(87.49 \mathrm{~Gb})$ sequencing data.

Before the genome assembly, we did the k-mer based genome survey analysis by using the GCE software (v1.0.0) (Liu et al., 2013) with the parameter: -k 17 -a 0 -o 0 -d 0 -t 8 -c 0.9 (Fig. S5 and Table S5). The primary genome was assembled using the supernova (v2.1.1) software (Weisenfeld et al., 2017), - maxreads $=700 \mathrm{M}$. Gaps were filled using GapCloser (Luo et al., 2012) and GapCloser stLFR (unpublished method) with default parameters. Finally, chromosome concatenation was performed using the $\mathrm{Hi}-\mathrm{C}$ generated data through the 3d-DNA pipeline (Dudchenko et al., 2017).

After the complete genome assembly, we performed the annotation pipeline. First, the Repeat Modeler (v1.0.11), LTR finder ( 11.0 .5$)$ and repeatscount ( $\mathrm{v} 1.0 .5)$ methods were used to identify de novo repeat motifs by ab initio modeling. The repeat motifs were added in the RepBase (Bao et al., 2015) library as known repeat elements. The Repeat Masker (Saha et al., 2008) was then performed to mask the assembly using the combined RepBase library.

Gene prediction was performed using homology-based, RNAseq based and de novo methods. Before the gene prediction, we masked repeat sequences by changing the $A / T / C / G$ to $N$ all across the genome (hard mask). We used Augustus, glimmerHMM, and SNAP for the de novo prediction (Table S7). For the homologybased approaches, Bombyx mori, Danaus plexippus, Drosophila melanogaster, and Spodoptera litura genomes were used for homology alignments using the TblastN. Moreover, gene sets were merged to form a non-redundant gene set with GLEAN for transcripts predicted by RNA-seq. Eventually, functional annotation of the proteins was performed using KEGG annotation.

\section{Comparative genomics analysis}

\section{Phylogenetic analysis}

Data for comparative genomics analysis contained Spodoptera frugiperda and other seven species from NCBI (https://www.ncbi.nlm. nih.gov/) including B. mori, Danaus plexippus, Drosophila melanogaster, Helicoverpa armigera, Manduca sexta, Plutella xylostella, and $S$. litura (Table S21). Based on the S. frugiperda-ref MAF alignment sequences from the other seven species, four degenerate 
sites for the 8 species were extracted to reconstruct the Maximumlikelihood tree using iqtree software with a bootstrap of 1000 .

\section{Genome synteny analysis of S. frugiperda and S. litura}

To identify the syntenic blocks in the whole genome, the coding sequences (CDSs) from $S$. frugiperda and $S$. litura were extracted using a custom python script, and the pairwise synteny was determined using LAST (Kielbasa et al., 2011). Tandem duplications and weak hits were then removed using module McScan (Python version) (Wang et al., 2012) from jcvi (https://github.com/tanghaibao/ jcvi). We extracted a subset of blocks using the options, "-minspan= 30 -simple". The high-quality syntenic karyotype was visualized by McScan from jcvi using the command, "python-m jcvi.graphics. karyotype seqids layout".

\section{Identifying gene families}

The specific gene families of eight species were identified using the HMMER3 (https://hmmer.janelia.org/) software which obtain $A B C$ domain (PF00005), COE domain (PF000135), Cytochrome P450 (CYP) domain (PF00067), GST domain (PF00043), UGT domain (PF00201), AOX domain (PF01315), CHIA domain (PF00704), $E P H X$ domain (PF06441), PIF domain (PF05970), PTCHD domain (PF02460), and PTP domain (PF00782), respectively. The Pfam-A dataset was downloaded from PFAM (https://pfam.janelia.org/) using the hmmscan program. The domain file was used as the first template to scan the gene family whereby the output genes were filtered out with an $\mathrm{E}$-value lower than $1 \mathrm{e}-10$. The filtered genes were used as second templates for another scanning of the gene families. Similarly, the second phase of output genes was filtered out with an E-value lower than $1 e-10$. Finally, the putative genes from the gene family were identified.

\section{Analyzing and defining the CYP gene family}

The putative CYP gene family of eight species databases was carried out using the HMMER3 software as in section "Identifying gene families". Since the KEGG annotation result on CYP gene was unclear, all the CYP genes of the eight species were annotated using the Kobas database (cabbage looper as annotation reference) by blast to reclassify the CYP gene subfamily into the following categories: CYP2, CYP3, CYP4, and mito-CYP. A large proportion of $C Y P$ for $S$. frugiperda was unannotated, therefore, we classified them as S.fr-specific CYP. According to the identity, the CYP sequences were clustered into 152 clusters by CD-HIT-EST (Fu et al., 2012) program with parameter "-c 0.95".

To confirm the accuracy of CYP scanning, the $S$. fr-specific CYP set and the CYP genes from $B$. mori and $S$. litura were subjected to the MEME program (Bailey et al., 2006) using the command "meme cyp.fasta-dna-o cyp-maxsize 2,000,000". The motif result and $10 \mathrm{bp}$ flanking sequences were uploaded to the website (https://weblogo. berkeley.edu/logo.cgi) to obtain the motif logo thereby displaying the conservation of the motif.

Identifying the positively selected genes (PSGs)

Based on the $S$. frugiperda-ref MAF alignment sequences from the B. mori, single-copy orthologous genes of S. frugiperda and B. mori were extracted to perform the positive selection analyses. A positive selection in FAW was tested using the PAML 4.9 (Yang 1997). The branch-site model for codon evolution was used with model $=2$, and NS sites (a parameter of PAML software $)=2$. Significance $(P<0.05)$ of the compared likelihood ratio tests (LRTs) was evaluated by $x^{2}$ tests using the PAML 4.9 (Yang, 1997). We assumed that the null distribution was a 50:50 mixture of a $X^{2}$ distribution with the point mass at zero. The QVALUE in R was used to correct for multiple testing. Finally, the PSG was identified with a cutoff of $P<0.05$.

\section{Transcriptome analyses}

Insects

The tested insects were collected from Yuanjiang, Yunnan Province of China (Table S20), the larvae were reared on fresh corn leaves without exposure to any pesticides for multiple generations, and the adults were provided with a $10 \%$ honey/water solution in a laboratory under the following controlled conditions of $(27 \pm 0.5)^{\circ} \mathrm{C},(70 \pm 5) \%$ $\mathrm{RH}$ and a photoperiod of $16 \mathrm{~h}: 8 \mathrm{~h}(\mathrm{~L}: \mathrm{D})$.

\section{Pesticide exposure}

Twenty-three pesticides commonly used in agricultural production were used in the study (Table S16), including ten single chemical pesticides, nine mixture of chemical pesticides and four biological pesticides. The third instar healthy larvae in the same size were selected for the experiment, and the larvae (3rd instar) were treated by adopted leaf-dipping method (Insecticide Resistance Action Committee, IRAC, 2019). In brief, fresh corn leaves (which had not been exposed to any pesticide) were washed clean and then naturally dried at room temperature. Thereafter, the leaves were cut into pieces $(2 \times 2 \mathrm{~cm})$ with scissors and dipped into different pesticide solutions (for treatments) or solvent (for control) for $10 \mathrm{~s}$ with gentle agitation and place to surface-dry on paper toweling, ensure the entire leaf surface is emerged equally and do not allow the leaves to wilt. After drying, the leaf discs treated with pesticide solutions at different concentrations or solvent for control were transferred into Petri dishes $(\phi=10 \mathrm{~cm})$ as insect food, and the Petri dishes were padded with moist filter paper at the bottom. Each treatment or control group had three replicates that contained 5 larvae each. Every $12 \mathrm{~h}$, the leaf pieces were replaced by fresh ones treated in the same way. After $48 \mathrm{~h}$ (72 $\mathrm{h}$ for biological pesticides) post exposure, three surviving larvae were collected for each treatment and put into liquid nitrogen for $2 \mathrm{~min}$, then frozen at $-80{ }^{\circ} \mathrm{C}$ in a sealed centrifuge tube for RNA extraction.

RNA isolation, transcriptome libraries preparation and sequencing

The RNA extraction kit (RNeasy Mini Kit, Qiagen) was used for total RNA isolation. The RNase-free agarose gel was performed to check for contamination. The RNA integrity and purity were measured using Agilent 2100 Bioanalyzer system (Agilent, United States) and NanoDrop Spectrophotometer (THERMO, United States), respectively. The extracted RNA was fragmented into $200-400$ bp and reverse transcribed to $\mathrm{cDNA}$ for library preparation. The libraries were prepared following the manufacturer's instructions using the BGISEQ-500 sequencing platform. Pair-end sequencing with $100 \mathrm{bp}$ 
in length was performed using the BGISEQ-500 sequencer with the processed libraries.

\section{Bioinformatics analysis for transcriptome data}

Raw data were initially processed using the Trimmomatic with the parameters of "ILLUMINACLIP:adapter.fa:2:30:4:1:true LEADING:3 TRAILING:3 SLIDINGWINDOW:5:15 MINLEN:50", and the SOAPfilter was used to filter reads with adaptors, reads with the proportion of Ns larger than $10 \%$, and reads with low-quality bases larger than $10 \%$ (Luo et al., 2012). Bridger software (v20141201) was used in de novo assembly of the transcriptomes. The redundancy was thereafter removed by TGICL (Pertea et al., 2003). The contigs were concatenated into scaffolds and further assembled to unigenes by clustering and removing redundancy. FPKM was calculated to estimate the expression level of the unigenes. The reads were mapped against the unigene library using Bowtie with the default parameters (Langmead 2010). The unique mapped reads were then selected to estimate the expression level using combining express. For the pesticide treatment samples, the DEG between every treatment with control and different combination of treatment with control was calculated. Also, the DEG of 78 samples were calculated using the edgeR and limma R packages. The $P$ value $<0.05$ were identified as DEGs. Unsupervised hierarchical clustering (Eisen et al., 1998) was carried out by cluster 3.0 software using uncentered Pearson correlation and complete linkage, and showed by Java Treeview software (Saldanha, 2004) into the "METHODS" section to describe the criterium of clustering.

\section{Identifying the strains and possible source of FAW in China}

Tpi gene was used as the DNA marker to identify strains of FAW from the USA, Africa and China in our study. There are eight strainspecific sites (TpiE4-129, TpiE4-144, TpiE4-165, TpiE4-168, TpiE4180, TpiE4-183, TpiE4-192, TpiE4-198) in the Tpi gene (Nagoshi et al., 2019), but the TpiE4-183 site is the most effective diagnostic marker to distinguish $\mathrm{C}$ or $\mathrm{R}$ strain. Therefore, we finally used this site to do the identification while other sites were also genotyped to assist the strain determination. We used our assembled genome as the reference, and mapped raw reads of all the 240 individuals (including 163 resequencing samples, 72 RNA-seq samples used for pesticide resistance analysis, one sample used for genome assembly survey, one sample used for stLFR genome assembly, one sample used for Hi-C sequencing and two RNA-seq samples used for gene annotation) to the reference genome by using the software BWA (Version: 0.7.10-r789) (Li et al., 2009). Duplicated reads, reads with mapping quality less than 30 and non-unique mapped reads were further filtered out to obtain the finally mapping result for the identification of genotypes of these eight sites. If the genotype is $C / C$, we identify it as the $C$ strain. Similarly, the $T / T$ and $\mathrm{C} / \mathrm{T}$ is identified as $\mathrm{R}$ strain and the hybrid strain.

\section{Resequencing analyses}

\section{Study populations}

We used 163 samples in six populations from America (39, A), South Africa (5, SA), Ethiopia (10, ET), Kenya (9, KE), Guangxi (56, GX),
Yunnan (44, YN), respectively, for the resequencing analyses (Figs. 4A, S8B and Table S20).

\section{Variant calling and SNP filtering}

Paired-end resequencing reads were mapped to our FAW assembly with BWA (Version: 0.7.10-r789) (Li and Durbin, 2009) using the default parameters. SAMtools (Version: 1.3.1) software ( $\mathrm{Li}$ et al., 2009) was used to convert mapping results into the BAM format and filter the unmapped and non-unique mapping reads. Duplicated reads were filtered using the Picard package (picard.sourceforge. net, Version: 2.1.1). The reads around the InDels were realigned using the Genome Analysis Toolkit (GATK, version 3.3-0-g37228af) in two steps (Mckenna et al., 2010). The first step involved using the RealignerTargetCreator package to identify regions that required realignment. In the second step, the InDelRealigner was used to realign the regions highlighted in the first step. This, therefore, generated a realigned BAM file for each accession.

The variation detection followed the best practice workflow recommended by GATK (Mckenna et al., 2010). The variants were called for each accession by the GATK HaplotypeCaller (Mckenna et al., 2010). A joint genotyping step for comprehensive variations union was performed on the gVCF files. In the filtering step, the SNP filter expression was set as $\mathrm{QD}<2.0|| \mathrm{MQ}<40.0$ || FS>100.0 || SOR >5.0 || MQRankSum<-12.5 || ReadPosRankSum<-8.0 || QUAL < 30 , and the InDel filter expression was set as $Q D<2.0$ \| ReadPosRankSum < 10.0 || InbreedingCoeff $<0.8$ || FS $>100.0$ || SOR $>5.0$ $\|$ QUAL $<30$. Specific insertions and deletions shorter than or equal to $40 \mathrm{bp}$ were considered. InDels and SNPs with none bi-allelic, > $40 \%$ missing calls, and MAF $<0.005$ were removed to yield the basic set. SNPs with MAF $<0.05$ (the core set) were further removed for phylogenetic tree structure, IBD calculation, PCA, and population structure analyses.

SNPs and InDels annotation was performed for FAW genome using the package ANNOVAR (Version: 2015-12-14) (Wang et al., 2010). The coverage of each accession against each chromosome of FAW genome was counted based on the aligned BAM file using SAMtools (Version: 1.3.1) software (Li et al., 2009). SNP density, InDel density, and total genetic diversity across each chromosome were counted with $100 \mathrm{~kb}$ sliding window using VCFtools software (v0.1.13) (Danecek et al., 2011).

\section{Population genetics analysis}

The whole-genome SNPs were used to construct the ML phylogenetic tree with 100 bootstraps using SNPhylo (Version: 20140701) (Lee et al., 2014). Spodoptera litura (Fabricius) was used to provide outgroup information at corresponding positions. The tool iTOL (https://itol.embl.de) was used to color the phylogenetic tree. For each group, the uncertain samples were discarded based on the phylogenetic tree for further analyses.

SNPs were pruned using PLINK (Version v1.90b3.38) (Purcell et al., 2007) with a window size of 50 SNPs (advancing 5 SNPs at a time) and an $r 2$ threshold of 0.5 . The PCA was performed through Genome-wide complex trait analysis (GCTA, version: 1.25.3) software (Yang et al., 2011). The first three eigenvectors were plotted. Population structure was analyzed using the ADMIXTURE (Version: 1.3) program (Alexander et al., 2009) with a block-relaxation 
algorithm. To explore the convergence of individuals, we predefined the number of genetic clusters $K$ from 2 to 8 and ran the crossvalidation error $(\mathrm{CV})$ procedure. Default methods and settings were used in the analyses.

The admixture graphs for geographically distinct groups were estimated using the TreeMix (Pickrell and Pritchard, 2012), using a maximum likelihood (ML) method based on a Gaussian model for allele frequency change. The topology changes of the $M L$ trees changes depending on the number of migration events $(\mathrm{m})$ allowed in the model. The values, $m=1$ to $m=5$ were used. The bootstrap values on the tree were based on 1,000 replicates. Arrows on the graph represented admixture events between different FAW populations.

\section{Demographic history reconstruction using PSMC}

The demographic history for FAWS from America ( $n=10$, three from $A A$, three from $A B$, two from $A C$, and two from $A D)$, Africa $(n=10$, four from $E T$, four from KE and two from $S A$ ) and China ( $n=10$, five from $\mathrm{YN}$ and five from GX) was inferred using a hidden Markov model approach following the pairwise sequentially Markovian coalescence (PSMC) (Liu and Hansen, 2017) based on SNP distribution. The parameters were set as follows: "-N25 -t15 -r5 -p 4+25*2+4 +6 ", "-d 13 -D 80", "-q20". A 0.25 year generation time and a mutation rate of $2.9 \times 10^{-9}$ mutations per nucleotide per year were used to convert the scaled times and population sizes into real times and sizes.

Selection signals analysis

SweeD (Version 3.3.1) (Pavlidis et al., 2013) was used to detect selective sweeps based on the CLR test. This detected signatures for artificial selection or a natural selection from each FAW group with grid number set as chromosome length/1000. CLR cut-off value of $A A, A B, A C, A D, E T, K E, S A, G X$ and $Y N$ were: 115.72, 2444.49, $89.40,265.15,136.09,162.07,280.32,77.53$ and 66.44, respectively.

\section{DATA AVAILABILITY}

The Raw sequencing data and the chromosome level genome assembly have been deposited to the CNSA (CNGB Nucleotide Sequence Archive) with accession CNP0000513 (https://db.cngb. org/cnsa/). The transcript and resequencing data have been deposited to the CNSA with accession CNP0001020. The genome data are also available through a BLAST webserver (https://159.226. 67.243/viroblast/fawmine.php) and genome database (https://159. 226.67.243:8080/fawmine).

\section{ABBREVIATIONS}

4DTv, Fourfold degenerate synonymous site; A, America; AA, America_A; $A B$, America_B; AC, America_C; $A D$, America_D; $A B C$, ATP-binding cassette; $A O X$, aldehyde oxidase; $B t$, Bacillus thuringiensis; CES, carboxylesterase; CHIA, chitinase acidic; CYP, cytochrome P450; DEG, differential expression gene; EPHX, epoxide hydrolase; EST, Expressed sequence tags; ET, Ethiopia; FAW, fall armyworm; GST, glutathione S-transferase; GX, Guangxi; Hi-C, high-throughput chromosome conformation capture; InDel, insertion and deletion; KE, Kenya; Kya, thousand years ago; LRT, likelihood ratio tests; MAF, minor allele frequency; ML, maximum likelihood; Mya, million years ago; PCA, principal component analysis; PIF, PIF1 5'-to-3' DNA helicase; PSG, Positively selected gene; PSMC, pairwise sequentially Markovian coalescen; $P T C H D$, patched domain containing; PTP, protein tyrosine phosphatase; SA, South Africa; SNP, single nucleotide polymorphism; stLFR, single tube long fragment read; UGT, UDP-glucuronosyl transferase; YN, Yunnan

\section{ACKNOWLEDGEMENTS}

This study was financially supported by the Yunnan Eco-friendly Food International Cooperation Research Center (YEFICRC) Project of Yunnan Provincial Key Programs (Grant No. 2019ZG009), the National Key R\&D Program of China (Grant No. 2019YFD0300101), the Guangdong Provincial Key Laboratory of Genome Read and Write (Grant No. 2017B030301011), the Key Research Program of the Chinese Academy of Sciences (KJZD-SW-L07), the Youth Innovation Promotion Association, CAS (No. 2016080) and KeyArea Research and Development Program of Guangdong Province (No. 2020B020224002). We thank the Guangdong Provincial Academician Workstation of BGI Synthetic Genomics, BGI-Shenzhen, Guangdong, China and the China National GeneBank for producing the sequencing data.

\section{AUTHOR CONTRIBUTIONS}

Huanming Yang, Le Kang, Jun Sheng, Yang Dong, Xin Liu and Huan Liu designed the research. Furong Gui, Huan Liu, Tianming Lan and Wei Guo collected the samples. Xiaofang Cheng, Furong Gui, Haorong Lu, Feng Chen, Guohai Hu, Ping Liu and Haimeng Li performed the DNA and RNA extraction and the library preparation. Tianming Lan, Yue Zhao, Dongming Fang, Guangyi Fan and Hongli Wang performed the data analysis. Ruoshi Hao, Yahong Li, Yaping Chen, Le Cheng, Shuqi He, Wei Dong, Bin Chen, Yongwei Zhang, Hanhong $\mathrm{Xu}$ and Fei Lin collected samples from China. Birhan Addisie Abate, Kassahun Tesfaye, Aschalew Lemma Demie, Meseret Destaw Bayeleygne and Dawit Tesfaye Degefu provided the FAW samples of Ethiopia. Bernard Slipper, Alisa Postma and Matthew Jackson provided the FAW samples of South Africa. Paul K Kuria and Zachary M Kinyua provided the FAW samples of Kenya. Fangneng Huang, Tongxian Liu and Yuan Jiang provided the FAW samples of America. Yang Dong, Yue Zhao, Tianming Lan, Furong Gui, Wei Guo, Dongming Fang, Pengcheng Yang and Sunil Kumar Sahu wrote the manuscript, Furong Gui, Yue Zhao, Tianming Lan, Yang Dong and Le Kang revised it critically. Huanming Yang, Jun Sheng and Le Kang secured funding. All authors read and approved the manuscript.

\section{COMPLIANCE WITH ETHICAL GUIDELINES}

Furong Gui, Tianming Lan, Yue Zhao, Wei Guo, Yang Dong, Dongming Fang, Huan Liu, Haimeng Li, Hongli Wang, Ruoshi Hao, Xiaofang Cheng, Yahong Li, Pengcheng Yang, Sunil Kumar Sahu, Yaping Chen, Le Cheng, Shuqi He, Ping Liu, Guangyi Fan, Haorong Lu, Guohai Hu, Wei Dong, Bin Chen, Yuan Jiang, Yongwei Zhang, Hanhong Xu, Fei Lin, Bernard Slipper, Alisa Postma, Matthew Jackson, Birhan Addisie Abate, Kassahun Tesfaye, Aschalew 
Lemma Demie, Meseret Destaw Bayeleygne, Dawit Tesfaye Degefu, Feng Chen, Paul K Kuria, Zachary M Kinyua, Tong-Xian Liu, Huanming Yang, Fangneng Huang, Xin Liu, Jun Sheng and Le Kang declare that they have no conflict of interest. This article does not contain any studies with human or animal subjects performed by the any of the authors.

\section{OPEN ACCESS}

This article is licensed under a Creative Commons Attribution 4.0 International License, which permits use, sharing, adaptation, distribution and reproduction in any medium or format, as long as you give appropriate credit to the original author(s) and the source, provide a link to the Creative Commons licence, and indicate if changes were made. The images or other third party material in this article are included in the article's Creative Commons licence, unless indicated otherwise in a credit line to the material. If material is not included in the article's Creative Commons licence and your intended use is not permitted by statutory regulation or exceeds the permitted use, you will need to obtain permission directly from the copyright holder. To view a copy of this licence, visit http:// creativecommons.org/licenses/by/4.0/.

\section{REFERENCES}

Adamczyk JJ Jr, Holloway JW, Leonard BR, Graves I (1997) Susceptibility of fall armyworm collected from different plant hosts to selected insecticides and transgenic Bt cotton. J Cotton Sci 1:21-28

Alexander DH, Novembre J, Lange K (2009) Fast model-based estimation of ancestry in unrelated individuals. Genome Res 19 (9):1655-1664

Bailey TL, Williams N, Misleh C, Li WW (2006) MEME: discovering and analyzing DNA and protein sequence motifs. Nucleic Acids Res 34(Web Server):W369-W373

Bao W, Kojima KK, Kohany O (2015) Repbase Update, a database of repetitive elements in eukaryotic genomes. Mob DNA 6:11

Bock KW (2016) The UDP-glycosyltransferase (UGT) superfamily expressed in humans, insects and plants: anima plant arms-race and co-evolution. Biochem Pharmacol 99(1):11-17

CABI (2019) Data sheet Spodoptera frugiperda (fall armyworm). Invasive Species Compendium. https://www.cabi.org/isc/ datasheet/29810\#94987198-9f50-41738bbd30bd93840e73? tdsourcetag=s_pcqq_aiomsg. Accessed 26 April 2019

Chang J, Yoon I, Lee J, Kim K, An J, Kim K (2010) Arsenic detoxification potential of aox genes in arsenite-oxidizing bacteria isolated from natural and constructed wetlands in the Republic of Korea. Environ Geochem Health 32(2):95-105

Chen W, Hasegawa DK, Kaur N, Kliot A, Pinheiro PV, Luan J, Stensmyr MC, Zheng Y, Liu W, Sun H et al (2016) The draft genome of whitefly Bemisia tabaci MEAM1, a global crop pest, provides novel insights into virus transmission, host adaptation, and insecticide resistance. BMC Biol 14:110

Cheng T, Wu J, Wu Y, Chilukuri RV, Huang L, Yamamoto K, Feng L, Li W, Chen Z, Guo H et al (2017) Genomic adaptation to polyphagy and pesticides in a major East Asian noctuid pest. Nat Ecol Evol 1(11):1747-1756
Daly H, Doyen JT, Purcell AH (1998) Introduction to insect biology and diversity. Oxford University Press, New York

Danecek P, Auton A, Abecasis G, Albers CA, Banks E, Depristo MA, Handsaker RE, Lunter G, Marth GT, Sherry ST et al (2011) The variant call format and VCF tools. Bioinformatics 27(15):21562158

Downing KJ, Leslie G, Thomson JA (2000) Biocontrol of the sugarcane borer Eldana saccharina by expression of the Bacillus thuringiensis cry $1 \mathrm{Ac7}$ and Serratia marcescens chiA genes in sugarcane-associated bacteria. Appl Environ Microbiol 66 (7):2804-2810

Dudchenko O, Batra SS, Omer AD, Nyquist SK, Hoeger M, Durand NC, Shamim MS, Machol I, Lander ES, Aiden AP et al (2017) De novo assembly of the Aedes aegypti genome using Hi-C yields chromosome-length scaffolds. Science 356(6333):92-95

Eisen MB, Spellman PT, Brown PO, Botstein D (1998) Cluster analysis and display of genome-wide expression patterns. Proc Natl Acad Sci USA 95:14863-14868

Erlandson MA (2009) Genetic variation in field populations of baculoviruses: mechanisms for generating variation and its potential role in baculovirus epizootiology. Virol Sin 24(5):458469

Farmer B (2019) Fall armyworm marches on as pest that devastated African crops spreads in Asia. Telegraph. https://www.telegraph. co.uk/news/2019/01/09/fall-armyworm-marches-pestdevastated-african-crops-spreads/. Accessed 26 April 2019

Fu L, Niu B, Zhu Z, Wu S, Li W (2012) CD-HIT: accelerated for clustering the next-generation sequencing data. Bioinformatics 28(23):3150-3152

Goergen G, Kumar PL, Sankung SB, Togola A, Tamò M (2016) First report of outbreaks of the fall armyworm Spodoptera frugiperda ( $\mathrm{J}$ E Smith) (Lepidoptera, Noctuidae), a new alien invasive pest in West and Central Africa. PLoS ONE 11(10):e165632

Gouin A, Bretaudeau A, Nam K, Gimenez S, Aury JM, Duvic B, Hilliou F, Durand N, Montagné N, Darboux I et al (2017) Two genomes of highly polyphagous lepidopteran pests (Spodoptera frugiperda, Noctuidae) with different host-plant ranges. Sci Rep 7:11816

Hayes JD, Pulford DJ (2008) The glutathione S-transferase supergene family: regulation of GST and the contribution of the isoenzymes to cancer chemoprotection and drug resistance Part I. Crit Rev Biochem Mol 30(6):445-520

Herraiz T, Guillen H, Aran V, Idle J, Gonzalez F (2006) Comparative aromatic hydroxylation and N-demethylation of MPTP neurotoxin and its analogs, $N$-methylated $\beta$-carboline and isoquinoline alkaloids, by human cytochrome P450 2D6. Toxicol Appl Pharmacol 216(3):387-398

Hinds WE, Dew JA (1915) The grass worm or fall army worm. Ala Agric Exp Stn Bull 186:61-92

Huang J, Liang X, Xuan Y, Geng C, Li Y, Lu H, Qu S, Mei X, Chen H, $\mathrm{Yu} T$ et al (2017) A reference human genome dataset of the BGISEQ-500 sequencer. GigaScience 6(5):1-9

larmarcovai G, Bonassi S, Botta A, Baan RA, Orsière T (2008) Genetic polymorphisms and micronucleus formation: a review of the literature. Mutat Res/Rev Mutat Res 658(3):215-233 
Insecticide Resistance Action Committee, IRAC (2019) IRAC susceptibility test methods series: method No. 018, version 3.4. www.irac-online.org. Accessed 13 Dec 2019

Jing DP, Guo JF, Jiang YY, Zhao JZ, Sethi A, He KL, Wang ZY (2019) Initial detections and spread of invasive Spodoptera frugiperda in China and comparisons with other noctuid larvae in cornfields using molecular techniques. Insect Sci 27(4):1-11

Juárez ML, Schöfl G, Vera MT, Vilardi JC, Murúa MG, Willink E, Hänniger S, Heckel DG, Groot AT (2014) Population structure of Spodoptera frugiperda maize and rice host forms in South America: are they host strains? Entomol Exp Appl 152(3):182199

Kakumani PK, Malhotra P, Mukherjee SK, Bhatnagar RK (2014) A draft genome assembly of the army worm, Spodoptera frugiperda. Genomics 104(2):134-143

Kielbasa SM, Wan R, Sato K, Horton P, Frith MC (2011) Adaptive seeds tame genomic sequence comparison. Genome Res 21 (3):487-493

Koenig C, Bretschneider A, Heckel DG, Grosse-Wilde E, Hansson BS, Vogel H (2015) The plastic response of Manduca sexta to host and non-host plants. Insect Biochem Mol Biol 63:72-85

Langmead B (2010) Aligning short sequencing reads with Bowtie. Curr Protoc Bioinform 32(11):1-14

Lee T, Guo H, Wang X, Kim C, Paterson AH (2014) SNPhylo: a pipeline to construct a phylogenetic tree from huge SNP data. BMC Genomics 15(1):162

Liu BH, Shi YJ, Yuan JY, Hu XS, Zhang H, Li N, Li ZY, Chen YX, Mu DS, Fan W (2013) Estimation of genomic characteristics by analyzing k-mer frequency in de novo genome projects. arXiv. https://www.researchgate.net/publication/255722390

Li H, Durbin R (2009) Fast and accurate short read alignment with Burrows-Wheeler transform. Bioinformatics 25(14):1754-1760

Li H, Handsaker B, Wysoker A, Fennell T, Ruan J, Homer N, Marth G, Abecasis G, Durbin R (2009) The sequence alignment/map format and SAM tools. Bioinformatics 25(16):2078-2079

Li XJ, Wu MF, Ma J, Gao BY, Wu QL, Chen AD, Liu J, Jiang YY, Zhai BP, Jason RE et al (2019) Prediction of migratory routes of the invasive fall armyworm in eastern China using a trajectory analytical approach. Pest Manag Sci 76(2):454-463

Lieberman-Aiden E, van Berkum NL, Williams L, Imakaev M, Ragoczy T, Telling A, Amit I, Lajoie BR, Sabo PJ, Dorschner $\mathrm{MO}$ et al (2009) Comprehensive mapping of long-range interactions reveals folding principles of the human genome. Science 326(5950):289-293

Liu S, Hansen MM (2017) PSMC (pairwise sequentially Markovian coalescent) analysis of RAD (restriction site associated DNA) sequencing data. Mol Ecol Resour 17(4):631-641

Luo R, Liu B, Xie Y, Li Z, Huang W, Yuan J, He G, Chen Y, Pan Q, Liu $Y$ et al (2012) SOAPdenovo2: an empirically improved memoryefficient short-read de novo assembler. GigaScience 1:18

Mallapur CP, Naik AK, Hagari S, Prabhu ST, Patil PK (2018) Status of alien pest fall armyworm, Spodoptera frugiperda (J.E. Smith) on maize in Northern Karnataka. J Entomol Zool Stud 6:432-436

Mckenna A, Hanna M, Banks E, Sivachenko A, Cibulskis K, Kernytsky A, Garimella K, Altshuler D, Gabriel S, Daly M et al (2010) The Genome Analysis Toolkit: a MapReduce framework for analyzing next-generation DNA sequencing data. Genome Res 20(9):1297-1303

Meagher RL, Nagoshi RN (2012) Differential feeding of fall armyworm (Lepidoptera: Noctuidae) host strains on meridic and natural diets. Ann Entomol Soc Am 105(3):462-470

Montezano DG, Specht A, Sosa-Gómez DR, Roque-Specht VF, Sousa-Silva JC, Paula-Moraes SV, Peterson JA, Hunt TE (2018) Host plants of Spodoptera frugiperda (Lepidoptera: Noctuidae) in the Americas. Afr Entomol 26:286-300

Morillo F, Notz A (2001) Resistance of Spodoptera frugiperda (Smith) (Lepidoptera: Noctuidae) to lambdacyhalothrin and methomyl. Entomotropica 16(2):79-87

Nagoshi RN, Goergen G, Plessis HD, van den Berg J, Meagher R (2019) Genetic comparisons of fall armyworm populations from 11 countries spanning Sub-Saharan Africa provide insights into strain composition and migratory behaviors. Sci Rep 9:8311

Nagoshi RN, Goergen G, Tounou KA, Agboka K, Koffi D, Meagher RL (2018) Analysis of strain distribution, migratory potential, and invasion history of fall armyworm populations in northern SubSaharan Africa. Sci Rep 8:3710

Nagoshi RN, Htain NN, Boughton D, Zhang L, Xiao Y, Nagoshi BY, Mota-Sanchez D (2020) Southeastern Asia fall armyworms are closely related to populations in Africa and India, consistent with common origin and recent migration. Sci Rep 10:1421

Nagoshi RN, Meagher RL (2004) Behavior and distribution of the two fall armyworm host strains in Florida. Fla Entomol 87(4):440449

Nam K, Gimenez S, Hilliou F, Blanco CA, Hänniger S, Bretaudeau A, Legeai F, Nègre N, d'Alencon E (2019) Adaptation by copy number variation increases pesticide resistance in fall armyworms. bioRxiv. https://www.biorxiv.org/content/https://doi.org/10. 1101/812958v1

Nam K, Nhim S, Robin S, Bretaudeau A, Nègre N, d'Alençon E (2018) Divergent selection causes whole genome differentiation without physical linkage among the targets in Spodoptera frugiperda (Noctuidae). bioRxiv. https://www.biorxiv.org/content/ https://doi.org/10.1101/452870v2

Nandakumar S, Ma H, Khan AS (2017) Whole-genome sequence of the Spodoptera frugiperda Sf9 insect cell line. Genome Announc 5(34):e00829-e917

Nowell RW, Elsworth B, Oostra V, Zwaan BJ, Wheat CW, Saastamoinen M, Saccheri IJ, van T Hof AE, Wasik BR, Connahs $\mathrm{H}$ et al (2017) A high-coverage draft genome of the mycalesine butterfly Bicyclus anynana. GigaScience 6(7):1-7

Pashley DP (1986) Host-associated genetic differentiation in fall armyworm (Lepidoptera: Noctuidae): a sibling species complex? Ann Entomol Soc Am 79(6):898-904

Pavlidis P, Ivković D, Stamatakis A, Alachiotis N (2013) SweeD: likelihood-based detection of selective sweeps in thousands of genomes. Mol Biol Evol 30(9):2224-2234

Pertea G, Huang X, Liang F, Antonescu V, Sultana R, Karamycheva S, Lee Y, White J, Cheung F, Parvizi B et al (2003) TIGR Gene Indices clustering tools (TGICL): a software system for fast clustering of large EST datasets. Bioinformatics 19(5):651-652

Pickrell J, Pritchard J (2012) Inference of population splits and mixtures from genome-wide allele frequency data. PLoS Genet 8 (11):e1002967 
Purcell S, Neale B, Todd-Brown K, Thomas L, Ferreira MAR, Bender D, Maller J, Sklar P, de Bakker PIW, Daly MJ et al (2007) PLINK: a tool set for whole-genome association and population-based linkage analyses. Am J Hum Genet 81(3):559-575

Saha S, Bridges S, Magbanua ZV, Peterson DG (2008) Empirical comparison of ab initio repeat finding programs. Nucleic Acids Res 36(7):2284-2294

Saldanha AJ (2004) Java Treeview-extensible visualization of microarray data. Bioinformatics 20:3246-3248

Santos-Amaya O, Rodrigues J, Souza T, Tavares C, Campos S, Guedes R, Pereira E (2016) Resistance to dual-gene Bt maize in Spodoptera frugiperda: selection, inheritance and cross-resistance to other transgenic events. Sci Rep 5:18243

Satoh T, Hosokawa M (2009) Carboxylesterases: structure, function and polymorphism. Biomol Ther 17(4):335-347

Sparks AN (1979) A review of the biology of the fall armyworm. Fla Entomol 62:82-86

Tay WT, Mahon RJ, Heckel DG, Walsh TK, Downes S, James WJ, Lee S, Reineke A, Williams AK, Gordon KHJ (2015) Insect resistance to Bacillus thuringiensis toxin cry2ab is conferred by mutations in an abc transporter subfamily a protein. PLoS Genet 11:e1005534

Triant DA, Cinel SD, Kawahara AY (2018) Lepidoptera genomes: current knowledge, gaps and future directions. Curr Opin Insect Sci 25:99-105

Unbehend $M$, Hänniger $S$, Vásquez $G M$, Juárez $M L$, Reisig $D$, McNeil JN, Meagher RL, Jenkins DA, Heckel DG, Groot AT (2014) Geographic variation in sexual attraction of Spodoptera frugiperda corn- and rice-strain males topheromone lures. PLoS ONE 9(2):e89255

Wang K, Li M, Hakonarson H (2010) ANNOVAR: functional annotation of genetic variants from high-throughput sequencing data. Nucleic Acids Res 38(16):e164

Wang O, Chin R, Cheng X, Wu MKY, Mao Q, Tang J, Sun Y, Anderson E, Lam HK, Chen D et al (2019) Efficient and unique cobarcoding of second-generation sequencing reads from long DNA molecules enabling cost-effective and accurate sequencing, haplotyping, and de novo assembly. Genome Res 29(5):798-808

Wang Y, Tang H, Debarry JD, Tan X, Li J, Wang X, Lee TH, Jin H, Marler B, Guo H et al (2012) MCScanX: a toolkit for detection and evolutionary analysis of gene synteny and collinearity. Nucleic Acids Res 40(7):e49
Weisenfeld NI, Kumar V, Shah P, Church DM, Jaffe DB (2017) Direct determination of diploid genome sequences. Genome Res 27 (5):757-767

Wenger JA, Cassone BJ, Legeai F, Johnston JS, Bansal R, Yates AD, Coates BS, Pavinato VAC, Michel A (2017) Whole genome sequence of the soybean aphid, Aphis glycines. Insect Biochem Mol 2017:102917

Weston DP, Poynton HC, Wellborn GA, Lydy MJ, Blalock BJ, Sepulveda MS, Colbourne JK (2013) Multiple origins of pyrethroid pesticide resistance across the species complex of a nontarget aquatic crustacean, Hyalella azteca. Proc Natl Acad Sci USA 110(41):16532-16537

Wu QL, He LM, Shen XJ, Jiang YY, Liu J, Hu G, Wu KM (2019) Estimation of the potential infestation area of newly-invaded fall armyworm Spodoptera frugiperda in the Yangtze River Valley of China. Insects 10:298

Xiao HM, Ye XH, Xu HX, Mei Y, Yang Y, Chen X, Yang YJ, Liu T, Yu YY, Yang WF et al (2020) The genetic adaptations of fall armyworm Spodoptera frugiperda facilitated its rapid global dispersal and invasion. Mol Ecol Resour 20(4):1050-1068

Yang J, Lee SH, Goddard ME, Visscher PM (2011) GCTA: a tool for genome-wide complex trait analysis. Am J Hum Genet 88(1):7682

Yang Z (1997) PAML: a program package for phylogenetic analysis by maximum likelihood. Comput Appl Biosci 13(5):555-556

You M, Yue Z, He W, Yang X, Yang G, Xie M, Zhan D, Baxter SW, Vasseur L, Gurr GM et al (2013) A heterozygous moth genome provides insights into herbivory and detoxification. Nat Genet 45 (2):220-225

Zhang L, Liu B, Zheng W, Liu C, Zhang D, Zhao S, Xu P, Wilson K, Withers A, Jones CM et al (2019) High-depth resequencing reveals hybrid population and pesticide resistance characteristics of fall armyworm (Spodoptera frugiperda) invading China. bioRxiv 2019: 813154. https://www.biorxiv.org/content/10.1101/813154v2

Zhang L, Liu B, Zheng W, Liu C, Zhang D, Zhao S, Li Z, Xu P, Wilson K, Withers A et al (2020) Genetic structure and insecticide resistance characteristics of fall armyworm populations invading China. Mol Ecol Resour. https://doi.org/10.1111/1755-0998.13219

Zhu F, Moural TW, Shah K, Palli SR (2013) Integrated analysis of cytochrome P450 gene superfamily in the red flour beetle, Tribolium castaneum. BMC Genomics 14(1):174 